\title{
The influence of developed cavitation on the flow of a turbulent shear layer
}

\author{
Claudia O. Iyer and Steven L. Ceccio \\ Department of Mechanical Engineering, University of Michigan, Ann Arbor, Michigan 49109-2121
}

(Received 28 January 2002; accepted 27 June 2002; published 28 August 2002)

\begin{abstract}
Developed cavitation in a shear layer was studied experimentally in order to determine the effect that the growth and collapse of cavitation have on the dynamics of shear flows. Planar particle imaging velocimetry (PIV) was used to measure the velocity field, the vorticity, strain rates, and Reynolds stresses of the flow downstream of the cavitating and noncavitating shear layer; the flow pressures and void fraction were also measured. The flow downstream of a cavitating shear flow was compared to the noncavitating shear flow. For cavitating shear layers with void fractions of up to $1.5 \%$, the growth rate of the shear layer and the mean flow downstream of the shear layer were modified by the growth and collapse of cavitation bubbles. The cross-stream velocity fluctuations and the Reynolds stresses measured downstream of the cavitating shear layer were reduced compared to the entirely noncavitating flow. This result is inconsistent with a scaling of the shear stress within the shear flow based on the mean flow. The decrease in the cross-stream fluctuations and Reynolds stresses suggests that the cavitation within the cores of strong streamwise vortices has decreased the coupling between the streamwise and cross-stream velocity fluctuations. (C) 2002 American Institute of Physics. [DOI: 10.1063/1.1501541]
\end{abstract}

\section{INTRODUCTION}

Cavitation has been observed to occur in turbulent shear flows when the local liquid pressure drops below the liquid vapor pressure and vapor bubbles form in the low-pressure cores of fluid vortices. Such "vortex cavitation" can occur within strong liquid jets, within regions of flow separation, and in the turbulent wakes of bluff objects (see Brennen ${ }^{1}$ for a recent review). Cavitating shear flows occur in the closure region of attached cavities and are associated with the formation of strong vortices downstream of a partial cavity (Laberteaux and Ceccio, ${ }^{2}$ Laberteaux et al., ${ }^{3}$ Gopalan and Katz, ${ }^{4}$ and Laberteaux and Ceccio ${ }^{5,6}$ ). The cavitating turbulent shear flow associated with attached cavitation plays an important role in the formation of cloud cavitation and in the processes that cause cavitation erosion (Kawanami et al., Callenaere et al. ${ }^{8}$.

Developed cavitating shear layers are complex multiphase flows. The continuous flow field is turbulent and characterized by relatively high Reynolds numbers (typically greater than 10000 based on integral length scales). The disperse (vapor) phase has a complex, nonspherical geometry and is often not in thermodynamic equilibrium with the continuous phase. Volume fractions can be relatively large (often much greater than $1 \%$ ). The vapor phase is often preferentially concentrated within the low-pressure cores of the strongest vortices, the sites of cavitation inception, and the presence of vapor in the shear layer can alter both the large-scale and small-scale flow processes. But, to date, experimental observations have not revealed which interactions between the vapor and the liquid flow fields constitute the dominant mechanisms responsible for overall flow modification. This question will be addressed by the present study through the experimental examination of a cavitating shear layer.

Large-scale spanwise vortical structures are an important feature of turbulent shear layers (Brown and Roshko, Winant and Brown ${ }^{10}$ ). Counter-rotating pairs of streamwise vortices can occur between the larger spanwise vortices (Bernal et al. ${ }^{11}$ Lasheras et al., ${ }^{12}$ and Lasheras and $\mathrm{Choi}^{13}$ ). With a reduction in free-stream pressure, the cores of the spanwise and streamwise vortices can fill with gas and vapor. Cavitating shear flows have been investigated by a number of researchers, and a review of the older literature is provided by Arndt. ${ }^{14}$ Katz $^{15}$ studied the cavitation inception within shear layers formed on axisymmetric bodies whose boundary layers underwent a laminar separation and subsequent turbulent reattachment. Katz and $\mathrm{O}^{\prime} \mathrm{Hern}^{16}$ and $\mathrm{O}^{\prime} \mathrm{Hern}^{17}$ examined cavitation inception and development in a plane shear layer for Reynolds numbers up to $2 \times 10^{6}$. Katz and $\mathrm{O}^{\prime} \mathrm{Hern}^{16}$ showed that cavitation inception occurs first within the streamwise vortices of the shear layer. The streamwise vortices are stretched between the spanwise vortices, resulting in a reduction in the vortex diameter and core pressure. A further reduction in the overall flow pressure will then lead to cavitation in the streamwise vortices. In a continuation of this study, O'Hern ${ }^{17}$ did not observe significant changes in the shear layer growth rate of a vortex roll-up process as the cavitation developed.

Cavitation forming in the turbulent wake of bluff objects has been investigated by many researchers, including Kermeen and Parkin, ${ }^{18}$ Young and Holl, ${ }^{19}$ Arndt ${ }^{20}$ Arndt and George, ${ }^{21}$ and Belahadji et al. ${ }^{22}$ Shedding of wake vortices can be altered by the development of cavitation in the vortex cores, and developed wake cavitation will typically increase the shedding frequency of the Karman vortices observed in the wake by up to $25 \%$. Belahadji et al. ${ }^{22}$ employed highspeed photography to investigate cavitation in the rotational structures of the turbulent wake behind a two-dimensional obstacle. They concluded that incipient cavitation in the 
wake acted as a passive agent of flow visualization, but developed cavitation actively altered the dynamics of the turbulent wake.

The presence of cavitation within a turbulent shear flow can potentially alter the dynamics of the flow through both local and global mechanisms. The presence of developed cavitation can lead to overall flow changes through decreases in mean flow density, increases in the overall flow volume, or modification of the mean pressure field. Cavitation can also modify the vortical flow locally. Belahadji et $a l^{22}$ have suggested that cavitation in the cores of vortices will affect the process of vortex stretching by the decoupling of the vortex strain and rotation rate. Gopalan and $\mathrm{Katz}^{4}$ demonstrated that significant flow-vapor interactions exist in the cavitating shear flow downstream of a partial cavity. And, Laberteaux and $\mathrm{Ceccio}^{5,6}$ showed how the growth and collapse of disperse cavitation bubbles led to the production of small-scale turbulence in the wake of partial cavities.

In the present study, we investigated how the development and collapse of cavitation within a plane shear layer alters the dynamics of the shear layer. The shear flow was examined from cavitation inception to full development. Planar particle imaging velocimetry (PIV) was used to examine the flow downstream of the cavitating shear layer, and flow pressures and void fractions were measured. We will show that the growth and collapse of cavitation within the shear layer altered the mean and fluctuating velocities of the flow downstream of the cavitating region.

\section{EXPERIMENTAL SETUP AND METHODS}

\section{A. Flow facility and test model}

Experiments were conducted in the Model Large Cavitation Channel (MLCC) in the Cavitation and Multiphase Flow Laboratory described by George and Ceccio. ${ }^{23}$ The MLCC is a recirculating water tunnel with a capacity of approximately $2.8 \mathrm{~m}^{3}$. Most of the lower leg has circular cross sections upstream and downstream of the impeller; the upper leg, including the contraction, test section, and diffuser, has a square cross section. The flat top of the MLCC allows low pressures in the test section without subvapor pressures and cavitation occurring in adjacent parts of the tunnel. As a result, the 6:1 contraction upstream of the test section is nonsymmetric, and internal flow straighteners are angled upward to make the velocity profile uniform within the test section. The rectangular test section has interior dimensions of 21.6 $\times 21.6 \times 118 \mathrm{~cm}$, with a flow area of $446 \mathrm{~cm}^{2}$ due to fillets in the corners. The four sides of the test section hold removable windows made of acrylic or aluminum. Before experiments, water is deaerated by circulation between the MLCC and a $2.27 \mathrm{~m}^{3}$ deaeration tank; the oxygen content of the water can be reduced to about $40 \%$ saturation in three hours. Flow in the MLCC is generated by an impeller in the bottom leg. The MLCC can operate at constant test section velocities of up to $15 \mathrm{~m} / \mathrm{s}$. The overall air content of the MLCC can be modified using a deaeration system. However, the nuclei content of the flow within the tunnel cannot be directly controlled. The oxygen content of the water was measured with a dissolved oxygen meter (Orion Model 810). Values of the oxygen content are reported at atmospheric pressure.

To create a shear layer, a sharp-edged plate spanning the full $21.6 \mathrm{~cm}$ width of the test section was placed vertically into the flow in a manner similar to that of Katz and O'Hern ${ }^{16}$ and O'Hern. ${ }^{17}$ The plate was $90 \mathrm{~mm}$ from the entrance to the test section, and mounted on a false floor to allow the boundary layer flow on the test section walls to pass beneath through a gap $12.5 \mathrm{~mm}$ tall. The false floor consists of a $58 \mathrm{~mm}$ tall and $710 \mathrm{~mm}$ long wedge. The height of the plate, $h$, is equal to $31 \mathrm{~mm}$. The top of the plate consists of a chamfered edge with a broken apex. The shear layer produced at the apex of the wedge flows into a diffuser with an angle of $9^{\circ}$. The pressure recovery in this diffuser is sufficient to cause desinence of the cavitating shear layer. A schematic diagram of the plate within the test section is shown in Fig. 1.

A differential pressure transducer (Mid-West Instrument Model 120) was used to measure the pressure between the entrance to the contraction and the wall above the apex of the plate. This pressure difference was used to determine the free-stream velocity, $U_{\infty}$, after calibration with a singlecomponent laser Doppler anemometry (LDA) system (Dantec DISA Type 55L90a) with the probe volume located midway between the apex of the plate and the top window of the test section. This tap was also used to measure the static pressure at the inlet of the test section with a Test Gauge by Ashcroft. Pressure was measured at this tap to determine the cavitation number, $\sigma=\left(P_{l}-P_{v}\right) / \frac{1}{2} \rho U_{\infty}^{2}$. The temperature of the flow was measured with a thermocouple and used to determine the liquid density, $\rho$, liquid kinematic viscosity, $\nu$, and vapor pressure, $P_{v}$. A second pressure tap was located downstream of the plate, and the differential pressure between the upstream and downstream taps was measured with a differential pressure transducer (Omega PX26-005DV). Moreover, dynamic pressure transducers were placed near these two pressure taps (PCB Piezotronics Model 102A05) to measure the fluctuating pressure component. Spectra of the fluctuating pressures were measured with a Stanford Research Systems SR770 FFT network analyzer. A bandwidth of $1 \mathrm{~Hz}$ was used over a range of $1024 \mathrm{kHz}$, and 1000 individual spectra were averaged. Side view and top view images of the shear layer were recorded with a Nikon $35 \mathrm{~mm}$ camera using flash photography and with a high-speed digital imaging system (Kodak EktaPro HS Motion Analyzer Model 4540mx).

\section{B. Void fraction measurement}

The average void fraction of the shear layer was measured using two different techniques. Light scattering was used to detect the passage of bubbles in a small probe volume. The LDA system was used to detect the passage of bubbles within its probe volume. As bubbles passed through the probe volume (with dimensions of $1.0 \mathrm{~mm}$ for the probe volume length and $0.12 \mathrm{~mm}$ for the beam waist diameter), a strong flash of laser light was detected by the photomultiplier tube. This signal saturated the tube, creating an electrical 


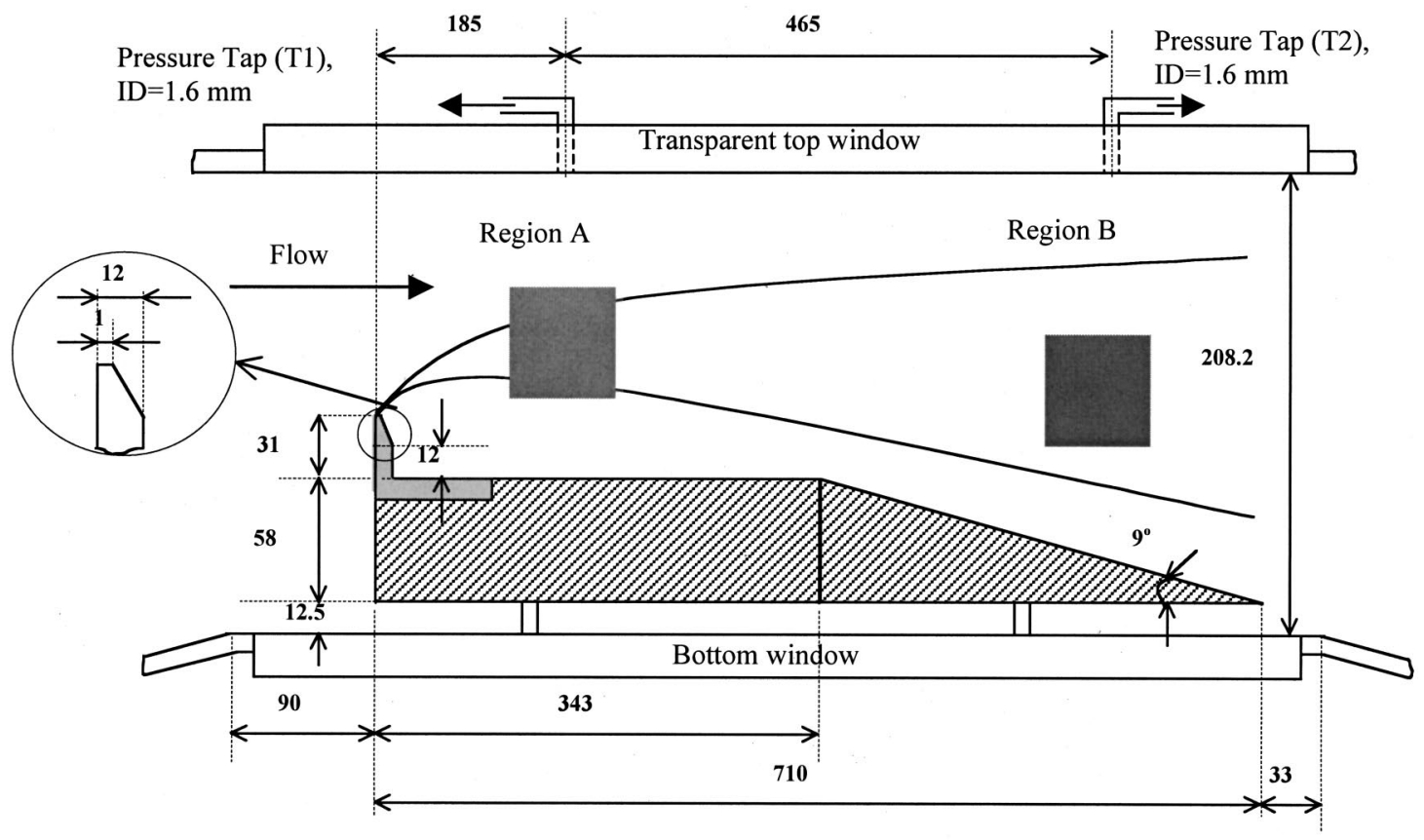

FIG. 1. Schematic diagram of the sharp-edged plate installed in the MLCO test section. The dimensions are in mm.

pulse with time width much longer that the actual bubble passage. Thus, the light scattering system could detect the passage of a bubble, but not the residence time within the probe volume. Instead, the residence time was measured with a high-speed digital imaging system (Kodak EktaPro HS Motion Analyzer Model 4540mx). The duration of the light pulses that resulted from the passage of bubbles within the probe volume was recorded and was typically $250 \mu \mathrm{s}$. Bubble event rates were measured for time intervals of $60 \mathrm{~s}$ and then averaged. With the bubble event rate, $f_{B}$, and residence time, $t_{B}$, the local void fraction, $\alpha$, can be calculated as

$$
\alpha=f_{B} t_{B} .
$$

The above equation provides a rough estimate of the void fraction. Second, a laser light sheet was used to illuminate a plane of the cavitating shear layer and digital images of the shear layer were recorded. (More details of the light sheet and camera setup are provided below.) The image of the shear layer contained images of the bubbles within the shear layer with some in focus and others out of focus. The image was thresholded and scaled to produce a binarized image that recovered only the in-focus bubbles. A pixel value of " 0 " was liquid and a value of " 1 " was gas. Five-hundred images were averaged to yield the overall average pixel value for the image and this was considered the void fraction. The void fraction values computed this way were consistent with the values obtained from light scattering, although the uncertainty of both measurements is relatively large.

\section{Particle imaging velocimetry}

The noncavitating and cavitating flows were examined with planar particle imaging velocimetry (PIV). PIV mea- surements were performed in region A, upstream, for noncavitating flows and in region B, downstream, for both noncavitating and cavitating flows, as shown in Fig. 1. Two frequency-doubled Quanta-Ray Spectra-Physics GCR-130 Nd:YAG lasers were used to create a double-pulsed light sheet in the test section. These lasers emit light pulses with $10 \mathrm{~ns}$ duration at $532 \mathrm{~nm}$ wavelength with a repetitive rate of $30 \mathrm{~Hz}$ and $30 \mathrm{~mJ} /$ pulse. The light sheet was approximately 1 $\mathrm{mm}$ thick and $100 \mathrm{~mm}$ wide and was placed parallel to the mean flow direction. Single-frame, double-pulsed images were recorded at $90^{\circ}$ to the light sheet plane with a Silicon Mountain Design SMD 4M4 digital camera that frames at 4 $\mathrm{Hz}$ and has a resolution of $2048 \times 2048$ pixels. The camera was mounted on an $X-Y$ traverse for easy translation along the test section. The light sheet making optics was also placed on a traverse that could be translated from region A to region $\mathrm{B}$ of the test section. The camera has an imageshifting feature whereby the image created by the first laser pulse is shifted vertically by a finite number of lines before the second image is recorded. This image shifting permits the introduction of a velocity bias in the double-pulsed image, thus eliminating ambiguity in the sign of the velocity. Images were acquired at $3 \mathrm{~Hz}$, and the time between light pulses, $\Delta t$, was measured within an accuracy of 50 ns with an HP 54620 A logic analyzer. The time separation, $\Delta t$, determines the maximum and minimum velocities that can be measured. The time separation, $\Delta t$, used in region A, upstream, was $21.6 \mu \mathrm{s}$ and in region $\mathrm{B}$, downstream, $53.6 \mu \mathrm{s}$. PIV measurements were made with a field of view of $5.5 \times 5.5 \mathrm{~cm}$. Spatial calibration of the image was performed by registering the image on a fixed object after a known motion on the traverse, and the traverse motion was measured to an accuracy of 50 $\mu \mathrm{m}$ over a span of $55000 \mu \mathrm{m}$. The flow was seeded with fluorescent latex particles $80 \mu \mathrm{m}$ in diameter with a specific 
gravity between 0.9 and 1.1. A filter was placed on the camera to block the green light of the lasers $(\lambda=532 \mathrm{~nm})$ and only allow the fluorescent return of the particles $(\lambda$ $=580 \mathrm{~nm}$ ). In this way, images of vapor bubbles could be excluded from the PIV image.

The double-pulsed images were interrogated using autocorrelation with the software package VISIFLOW by AEA Technology. An interrogation region of $128 \times 128$ pixels was used with an overlap of $50 \%$, resulting in a resolution of 1.7 $\mathrm{mm}$ with a field of view of $5.5 \times 5.5 \mathrm{~cm}$. There were typically 8-15 particle pairs within each interrogation region. With a time separation of $21.6 \mu \mathrm{s}$ in region A and $53.6 \mu \mathrm{s}$ in region $\mathrm{B}$, a velocity of $5 \mathrm{~m} / \mathrm{s}$ would correspond to a particle displacement of four pixels and ten pixels, respectively. The number of bad vectors in each image was typically $5 \%$ or less and interpolation was used to replace them. Each processed image yielded $32 \times 32$ instantaneous local velocity vectors in the plane of interest. The associated vorticity and strain-rate distributions were computed from the twodimensional velocity field using centered finite differences:

$$
\begin{aligned}
\omega_{z}(i, j)=\frac{\partial v}{\partial x}-\frac{\partial u}{\partial y}= & \frac{v(i+1, j)-v(i-1, j)}{2 \Delta x} \\
& -\frac{u(i, j+1)-u(i, j-1)}{2 \Delta y}, \\
S_{x y}(i, j)=\frac{\partial v}{\partial x}+\frac{\partial u}{\partial y}= & \frac{v(i+1, j)-v(i-1, j)}{2 \Delta x} \\
& +\frac{u(i, j+1)-u(i, j-1)}{2 \Delta y},
\end{aligned}
$$

where $u$ and $v$ are the horizontal and vertical velocity components, $i$ and $j$ are the horizontal and vertical indices, and $\Delta x$ and $\Delta y$ are the horizontal and vertical grid spacing. Up to 1000 images were averaged to find the average and fluctuating component of these quantities.

\section{Experimental uncertainty}

The estimated uncertainty in the measured mean pressures and velocity is $\pm 2 \%$. The temperature was accurate to within $\pm 0.2^{\circ} \mathrm{C}$. This leads to an uncertainty in the cavitation number of $\pm 6 \%$. The uncertainty of the PIV velocity is related to the uncertainty in the spatial and temporal measurements for the system. The uncertainty of the spatial measurement is related to the magnification of the image area, the particle displacement, and the size of the PIV autocorrelation interrogation area. For a speed of $5 \mathrm{~m} / \mathrm{s}$, the average particle displacement was 10 pixels. The displacements are known to subpixel resolution of \pm 0.2 pixels. This yields an uncertainty in the displacements of around $\pm 2 \%$. The uncertainty is larger for the cross-stream velocities since they are on the order of $0.5 \mathrm{~m} / \mathrm{s}$ or less. The uncertainty in the spatial calibration is $0.1 \%$ (based on the traverse accuracy of $50 \mu \mathrm{m}$ over a span of $55000 \mu \mathrm{m})$. The time separation between successive laser light pulses is measured very accurately within $50 \mathrm{~ns}$, which makes the uncertainty of the measured time interval less than $\pm 0.1 \%$. Thus, we estimate that the uncertainty in the measured velocity is approximately
$1 \%-2 \%$ for the streamwise ( $u$ component) of the velocities, and $10 \%-20 \%$ for the cross-stream ( $v$ component) of the velocities. The uncertainty in the measurement of the average void fraction is about $25 \%$ of the reported average value.

The smallest resolvable velocity fluctuation, $\Delta u / U_{\mathrm{fs}}$, is \pm 0.02 based on the minimum resolution of the PIV processing of \pm 0.2 pixels. Here $N=1000$ images were averaged to achieve a higher level of precision, and the resulting uncertainty is approximated by $\Delta u / U_{\mathrm{fs}} \cdot(1 / \sqrt{N}) \sim \pm 0.001$. Differences of less than \pm 0.002 are within the uncertainty of the measurement. If the integral length scale of the flow is $\sim 2 \delta=L_{I}$, the spatial resolution of the PIV images is $\sim 0.03 \cdot L_{I}$. This compares with an estimate of the Taylor length scale $L_{\lambda} \approx L_{I} \sqrt{\left(\nu / k^{1 / 2} L_{I}\right)} \approx L_{I} \sqrt{\left(\nu / 0.2 \cdot U_{\mathrm{fs}} \cdot 2 \delta\right)}$ $\approx 0.003 \cdot L_{I}$, where the turbulent kinetic energy, $k$, is approximated with $k^{1 / 2} \approx \sqrt{\overline{u^{\prime} u^{\prime}}} \approx 0.2 U_{\text {fs }}$. Consequently, these measurements do not spatially resolve the smallest turbulent length scales of the flow and the implication for not resolving all scales is that we will likely underestimate the local turbulent intensity.

The smallest resolvable Reynolds stress, $\Delta u \Delta u / U_{\mathrm{fs}}^{2}$, is \pm 0.04 , based on the minimum resolution of the PIV processing of \pm 0.2 pixels. The $N=1000$ images were averaged to achieve a higher level of precision, and the resulting uncertainty is approximated by $\Delta\left(\Delta u \Delta u / U_{\mathrm{fs}}^{2}\right)=\Delta u \Delta u / U_{\mathrm{fs}}^{2} \cdot(1 /$ $\sqrt{N}) \sim \pm 0.001$. Differences of less than \pm 0.002 are within the uncertainty of the measurement.

\section{RESULTS AND DISCUSSION}

\section{A. The non-cavitating flow of the shear layer in region $A$}

PIV was used to examine the noncavitating flow of the shear layer near the origin of the shear layer in region $\mathrm{A}$. The free-stream velocity measured above the shear layer was $U_{\infty}=9 \mathrm{~m} / \mathrm{s}$, giving a Reynolds number based on the plate height, $h=31 \mathrm{~mm}$, of $\mathrm{Re}=2 h U_{\infty} / \nu=5.56 \times 10^{5}\left(\mathrm{O}^{\prime} \mathrm{Hern}^{17}\right.$ reported data for $\left.\operatorname{Re}=2 h U_{\infty} / \nu=9.57 \times 10^{5}\right)$. The free-stream pressure was sufficiently high to prevent cavitation ( $\sigma$ $=2.3$ ). Figure 2 shows (a) the mean in-plane velocity, $\bar{u}$ $-U_{\text {Mean,A }}$, (b) the mean vorticity, $\omega_{z}$, (c) the mean strain rate, $S_{x y}$, and (d) the mean Reynolds stress, $-\overline{u^{\prime} v^{\prime}} / U_{\text {Mean, } \mathrm{A}}^{2}$. Here $U_{\text {Mean,A }}=3.86 \mathrm{~m} / \mathrm{s}$ and is the average velocity determined by averaging all $32 \times 32$ vectors of the mean velocity field of region A. 700 individual vector realizations were averaged. As expected, high levels of vorticity, strain rate, and Reynolds stresses are observed in the middle of the shear layer.

The data from Fig. 2 was used to determine the nondimensional mean-axial velocity profiles $(\bar{u}$ $\left.-U_{\text {Bottom,A }}\right) /\left(U_{\text {Top,A }}-U_{\text {Bottom,A }}\right)$. These are plotted against the nondimensional vertical distance, $y / \delta$, at three crosssections of region A: $x / h=5.53, x / h=5.96$, and $x / h=6.4$, in Fig. 3. The $y$ axis was first shifted by $\delta$ such that the origin of the $y$ axis corresponds to the point where $(\bar{u}$ $\left.-U_{\text {Bottom,A }}\right) /\left(U_{\text {Top,A }}-U_{\text {Bottom,A }}\right)=0.5$ and then the $y$ values were nondimensionalized by $\delta=26.2 \mathrm{~mm}$, corresponding to approximately half of the local shear layer thickness. Here $U_{\mathrm{Top}, \mathrm{A}}=7.0 \mathrm{~m} / \mathrm{s}$ and $U_{\mathrm{Bottom}, \mathrm{A}}=0.5 \mathrm{~m} / \mathrm{s}$ are the average ve- 


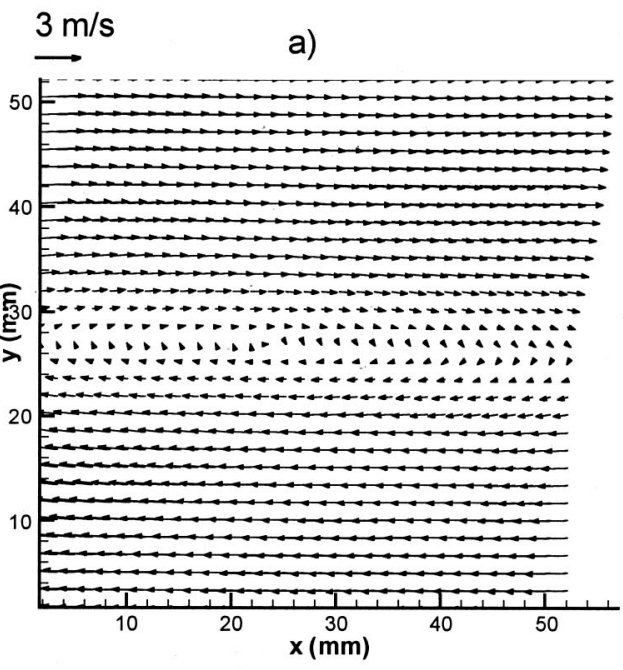

c)

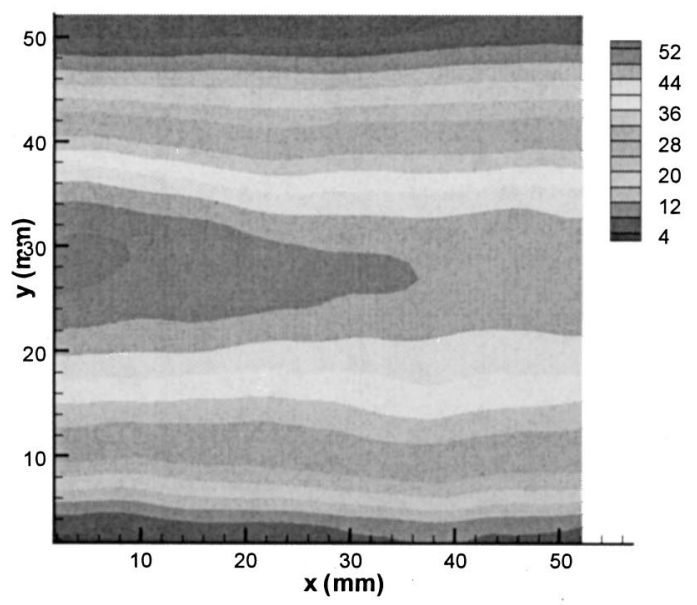

b)

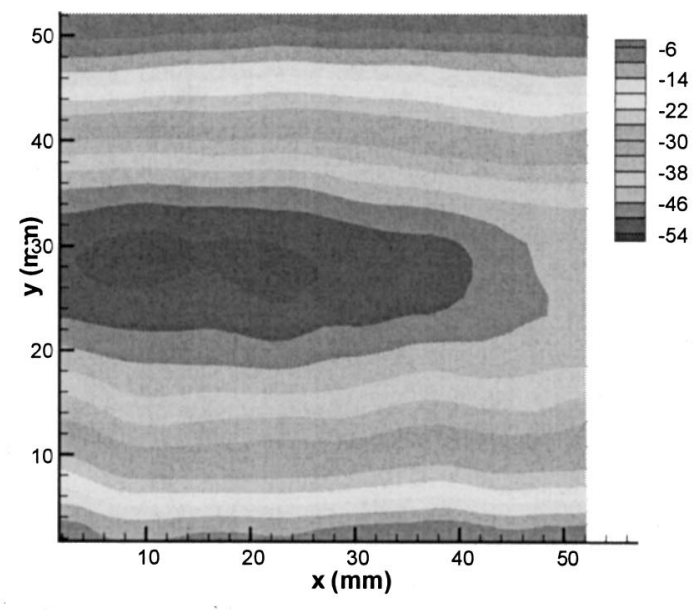

d)

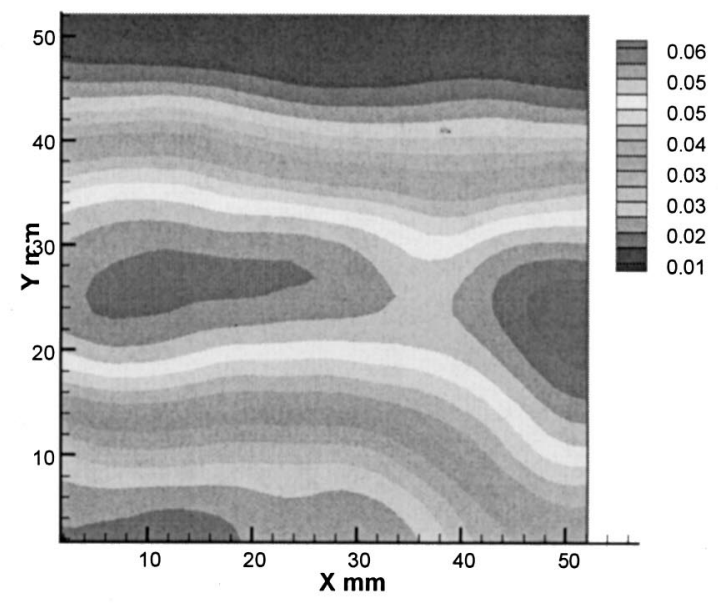

FIG. 2. Average noncavitating flow in region A presenting (a) mean velocity, $\bar{u}-U_{\mathrm{Mean}, \mathrm{A}}(\mathrm{m} / \mathrm{s}),(\mathrm{b})$ vorticity, $\omega_{z}(1 / \mathrm{s}),(\mathrm{c})$ strain rate, $S_{x y}(1 / \mathrm{s})$; and $(\mathrm{d})$ the Reynolds stresses $-u^{\prime} v^{\prime} / U_{\text {Mean, } \mathrm{A}}^{2}$. The flow is from left to right. Here 1000 individual images were averaged.

locities of the high- and low-speed free-stream flows measured at the top and bottom boundary of the PIV images in region A. Three averaged velocity profiles across the shear layer are shown for three distances measured from the vertex of the plate. In this figure the downstream distance $x$ was nondimensionalized by the plate height $h$. (This length scale was similarly chosen by $\mathrm{O}^{\prime} \mathrm{Hern}^{17}$ and it is on the order of the vortex spacing in region A.)

The velocity profiles indicate that the shear layer is close to achieving a similarity condition. Using the average shear layer thickness, $2 \delta$, in region A and the velocity difference, ( $U_{\text {Top,A }}-U_{\text {Bottom, } \mathrm{A}}$ ), the average Reynolds number is calculated as $\operatorname{Re}_{\delta}=2 \delta\left(U_{\text {Top, } \mathrm{A}}-U_{\text {Bottom, } \mathrm{A}}\right) / \nu=3.35 \times 10^{5}$. Wygnanski and Fiedler ${ }^{24}$ used hot-wire probes to examine a twodimensional, incompressible shear layer of air forming downstream of a backward facing step. This flow geometry is analogous to that of the present study. Their flow had a local Reynolds number on the order of $\operatorname{Re}_{\delta} \approx 10^{4}$ after the shear layer achieved similarity. Figure 4 presents the distribution of the nondimensional velocity fluctuation profiles, $\overline{u^{\prime} u^{\prime}} /\left(U_{\text {Top }, \mathrm{A}}-U_{\text {Bottom, } \mathrm{A}}\right)^{2}$ and $\overline{v^{\prime} v^{\prime} /}\left(U_{\text {Top }, \mathrm{A}}-U_{\text {Bottom }, \mathrm{A}}\right)^{2}$, at

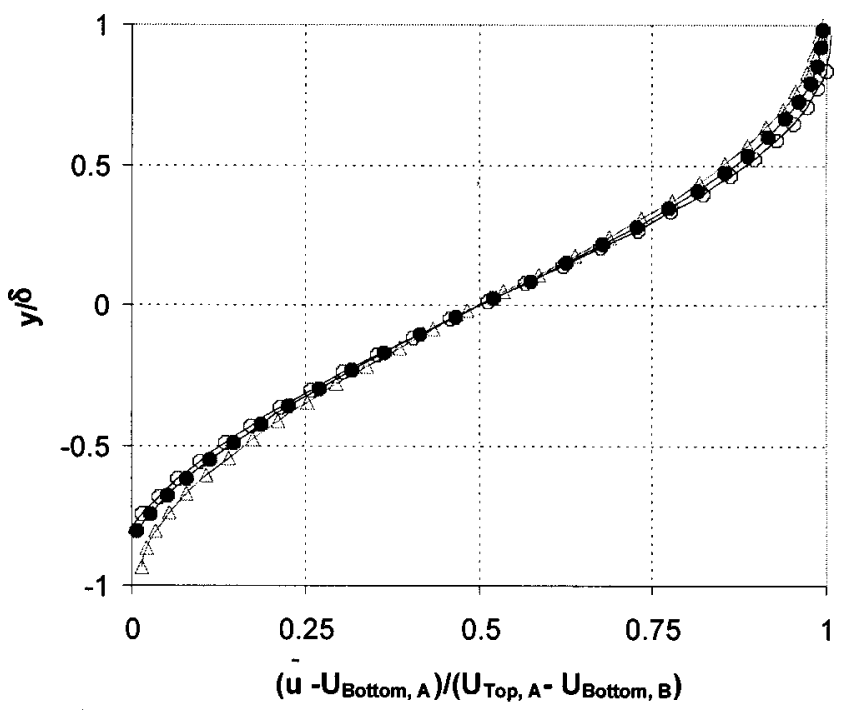

FIG. 3. Mean axial velocity profiles in region A derived from the data in Fig. 2. Here $\left(\bar{u}-U_{\text {Bottom }, \mathrm{A}}\right) /\left(U_{\text {Top, } \mathrm{A}}-U_{\text {Bottom }, \mathrm{A}}\right)$ is plotted across the shear layer for three downstream positions, $x / h$. The symbols $\bigcirc, \boldsymbol{\bullet}$, and $\triangle$ indicate $x / h=5.53,5.97$, and 6.40 , respectively. 


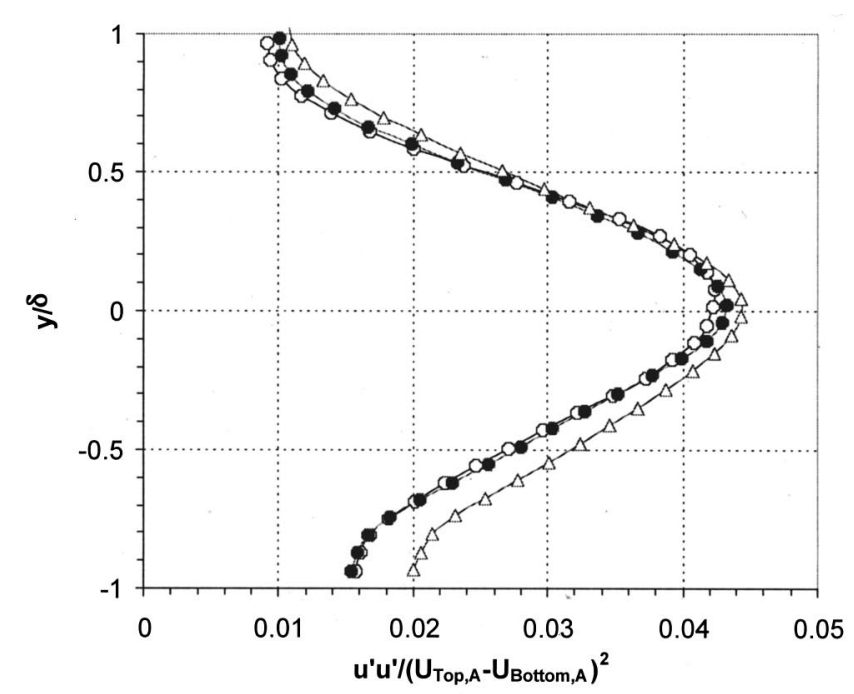

(a)

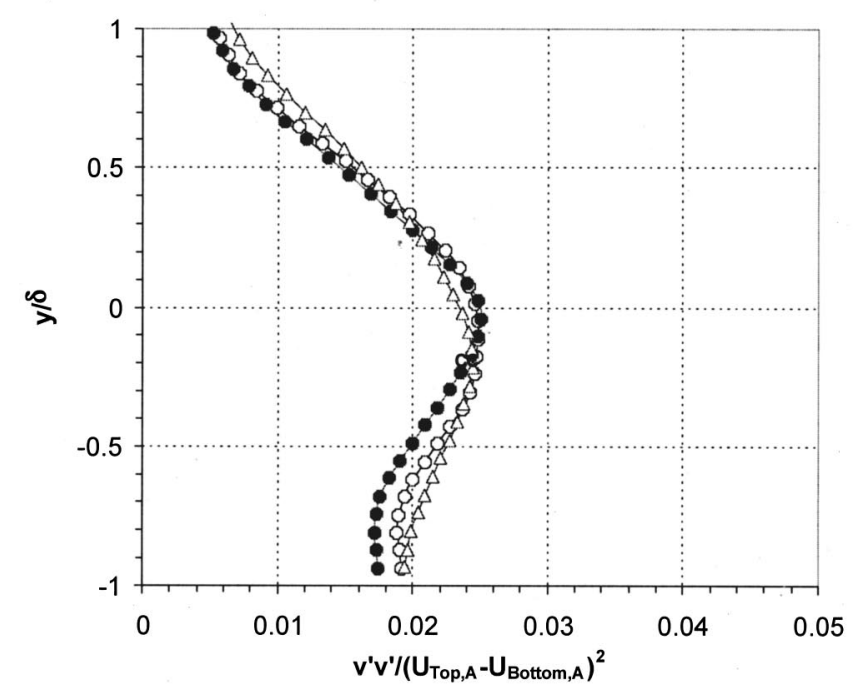

(b)

FIG. 4. Velocity fluctuation profiles (a) $u^{\prime} u^{\prime} /\left(U_{\mathrm{Top}, \mathrm{A}}-U_{\mathrm{Bottom}, \mathrm{A}}\right)^{2}$ and (b) $v^{\prime} v^{\prime} /\left(U_{\text {Top,A }}-U_{\text {Bottom,A }}\right)^{2}$ in region A derived from the data in Fig. 2 for three downstream positions, $x / h$. The symbols $\bigcirc, \boldsymbol{\bullet}$, and $\triangle$ indicate $x / h$ $=5.53,5.97$, and 6.40 , respectively.

three cross-sections. The maximum velocity fluctuation amplitude is $4.4 \%$, which qualitatively agrees with a maximum value of $18 \%$ reported by Wygnanski and Fiedler ${ }^{24}$ for the nondimensional root-mean-square velocity fluctuations. It should also be noted that the turbulence level does not tend to zero outside of the shear layer, and this suggests that the free-stream turbulence levels are relatively high (on the order of $2 \%$ of the free-stream), even with the flow conditioning and 6:1 contraction. This indicates that the shear layer in region $\mathrm{A}$ is reasonably two-dimensional and has becomes self-similar before reaching the diffuser in region $\mathrm{B}$.

\section{B. Examination of the cavitating shear layer}

While maintaining the free-stream velocity at $10 \mathrm{~m} / \mathrm{s}$, the free-stream pressure was reduced, and cavitation was observed in region A. The inception cavitation number was approximately $\sigma=0.65$ for a dissolved oxygen content of 3 ppm and $\sigma=0.57$ for a dissolved oxygen content of $1.7 \mathrm{ppm}$. O'Hern ${ }^{17}$ reported a cavitation inception index of $\sigma=1.1$ for a dissolved air content of $3.6 \mathrm{ppm}$ and $\sigma=1.4$ for an air content of 7-12 ppm. The extent of cavitation developed at 3 ppm is similar to that reported by O'Hern. ${ }^{17}$ (See Yu and $\mathrm{Ceccio}^{25}$ for a discussion of the relationship between dissolved oxygen content and dissolved air content.) In both the present experiment and those of $\mathrm{O}^{\prime} \mathrm{Hern},{ }^{17}$ the free-stream nuclei content was not controlled or measured. These differences in inception indices between the work of O' $\mathrm{Hern}^{17}$ and the present work can be partially explained by the freestream nuclei content. However, our method used to call inception in the present work is "visual calls" and it leads to a delayed cavitation call compared to the method of O'Hern.

Figure 5 presents side-view photographs, taken under stroboscopic light, of different levels of cavitation in the shear layer (with an oxygen content of $2.7 \mathrm{ppm}$ ). Figure 5(a) shows that inception cavitation occurs in the form of long, thin streamwise cavities oriented at about $45^{\circ}$ to the mean flow, visualizing the low-pressure cores of streamwise vortices of the shear layer that form between adjacent spanwise vortices. At lower cavitation numbers, the entire turbulent structure of the shear layer can be visualized by cavitation within both streamwise and spanwise vortices, as seen in Figs. 5(b)-5(e). At the lowest cavitation number [Fig. 5(e)] the recirculating region beneath the cavity becomes filled with a bubbly mixture. These observations of incipient and developed shear layer cavitation are consistent with those of Katz and O'Hern ${ }^{16}$ and O'Hern. ${ }^{17}$ Figure 6 presents a time series of the developed cavitating shear layer formed behind the sharp-edged plate $\left(\sigma=0.52, \quad U_{\infty}=9.0 \mathrm{~m} / \mathrm{s}\right.$, and $c_{\mathrm{O}}$ $=2.7 \mathrm{ppm}$, where $c_{\mathrm{O}}$ is the oxygen content). Such highspeed video images were analyzed to determine the shear layer growth rate and vortex spacing.

O'Hern ${ }^{17}$ observed that the growth of the cavitating shear layer was little changed from that of the noncavitating shear layer, and a similar result was found in the present study. Figure 7 shows a plot of the dimensionless shear layer thickness, $\delta^{*} / h$, versus the dimensionless downstream distance, $x / h$. The growth rate, $\delta^{*} / h$, had a slope of 0.25 , and this compares well to the value of 0.23 found by O'Hern ${ }^{17}$ for a cavitating shear layer, and to the value of 0.22 found by Wygnanski and Fiedler. ${ }^{24}$ The spacing of the spanwise cavitating vortices increases with an increasing downstream distance. Figure 8 shows the average spanwise vortex spacing, $l$, as a function of the downstream distance, $x$, for two cavitation numbers. The spanwise vortex spacing constant is defined as $l / x$, and this was found to be 0.47 for $\sigma=0.45$ and 0.51 for $\sigma=0.57$. A value of 0.50 was found by $\mathrm{O}^{\prime} \mathrm{Hern}^{17}$ for a shear flow with developed cavitation. Bernal et al., ${ }^{11}$ Hernan and Jimenez, ${ }^{26}$ and Jimenez ${ }^{27}$ found values of 0.56 for noncavitating turbulent plane shear layer with $\operatorname{Re}_{\delta}$ $>10^{4}$. The values found for the cavitating shear layer are equivalent to those measured for the noncavitating layer, within the uncertainty of the measurement.

Figure 9 presents the average spacing and diameter of the spanwise vortices, and the length of the streamwise vortices as a function of cavitation number. These quantities were averaged over the entire field of view (about $10 \mathrm{~cm}$ in 


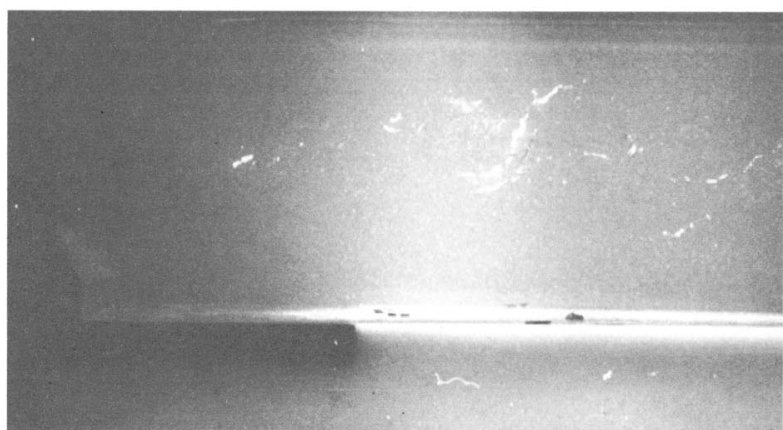

(a)

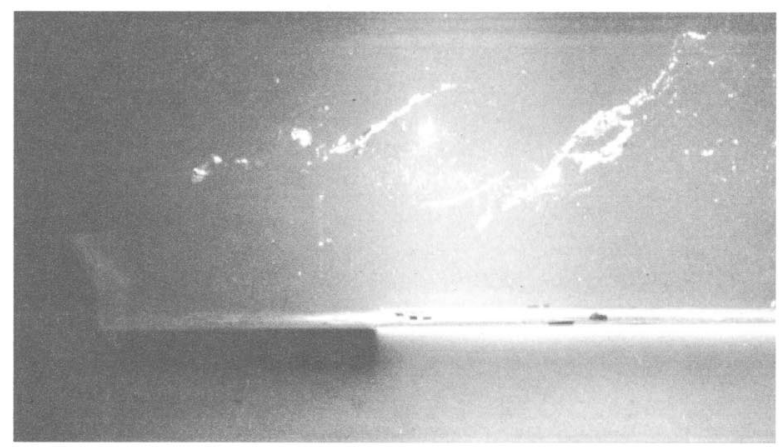

(b)

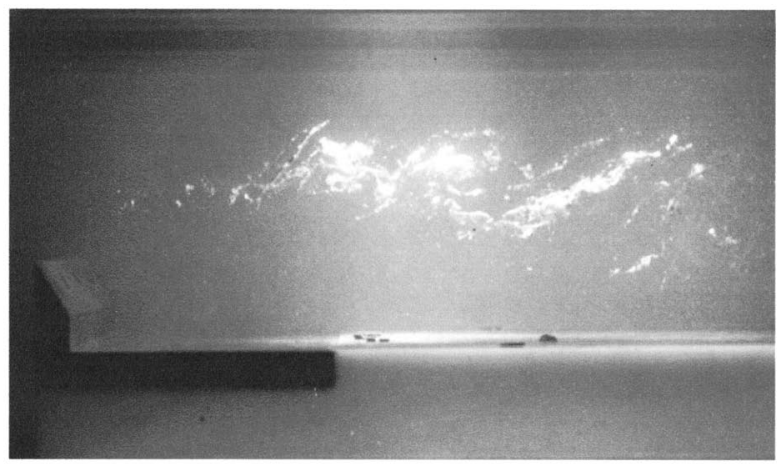

(c)

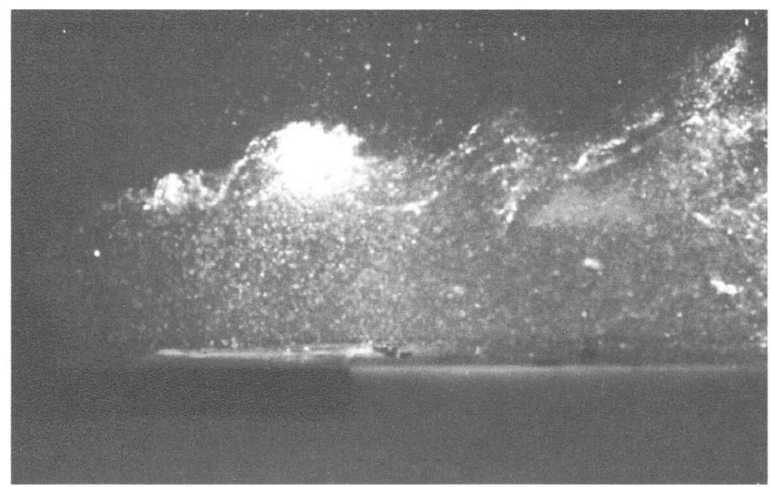

(d)

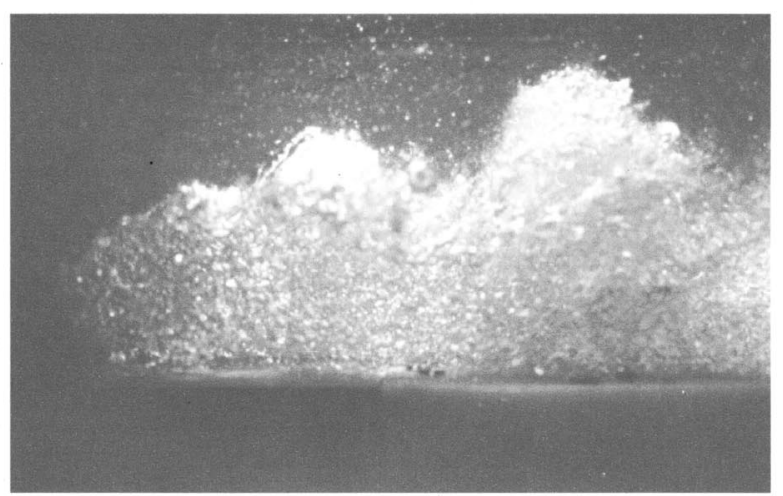

(e)

FIG. 5. (a) Photograph of incipient cavitation for $\sigma=0.65, U_{\infty}=9.0 \mathrm{~m} / \mathrm{s}$. (b) Photograph of incipient cavitation for $\sigma=0.57, U_{\infty}=9.0 \mathrm{~m} / \mathrm{s}$. (c) Photograph of incipient cavitation for $\sigma=0.50, U_{\infty}=9.0 \mathrm{~m} / \mathrm{s}$. (d) Photograph of incipient cavitation for $\sigma=0.41, U_{\infty}=9.0 \mathrm{~m} / \mathrm{s}$. (e) Photograph of incipient cavitation for $\sigma=0.33, U_{\infty}=9.0 \mathrm{~m} / \mathrm{s}$

the streamwise direction and $15 \mathrm{~cm}$ in the spanwise direction) for the growing shear layer. The lengths in this figure were nondimensionalized by $2 \delta$, where $\delta=26.2 \mathrm{~mm}$ is half the shear layer thickness measured in region A. Only small changes are seen with a variation in the cavitation number. In Fig. 9, the average spacing of the spanwise vortices, 1, decreases with decreasing cavitation number as the number of vortices observed increases. In the same figure, a slight increase in the diameter of the spanwise vortices and the length of the streamwise vortices is observed, although these changes are well within the range of uncertainty. Note that there is a slight increase in the diameter and the length of these vortices for $\sigma=0.50$ as the cavitation number is lowered to 0.45 .
If a noncavitating streamwise vortex is simply modeled as a Rankine vortex with core radius, a, the pressure within the core, $P_{c}$, is given by

$$
P_{\infty}-P_{C}=\rho\left(\frac{\Gamma_{S}}{2 \pi a}\right)^{2}
$$

where $\Gamma_{s}$ is the strength of the vortex. At the point of cavitation inception in the vortex, the core pressure reaches the vapor pressure, $P_{c}=P_{v}$, assuming that the water is sufficiently populated with cavitatable nuclei (i.e., the water is "weak"). In this case, the vortex strength would be given by

$$
\Gamma_{S}=2 \pi a U_{\infty} \sqrt{\sigma_{i}},
$$



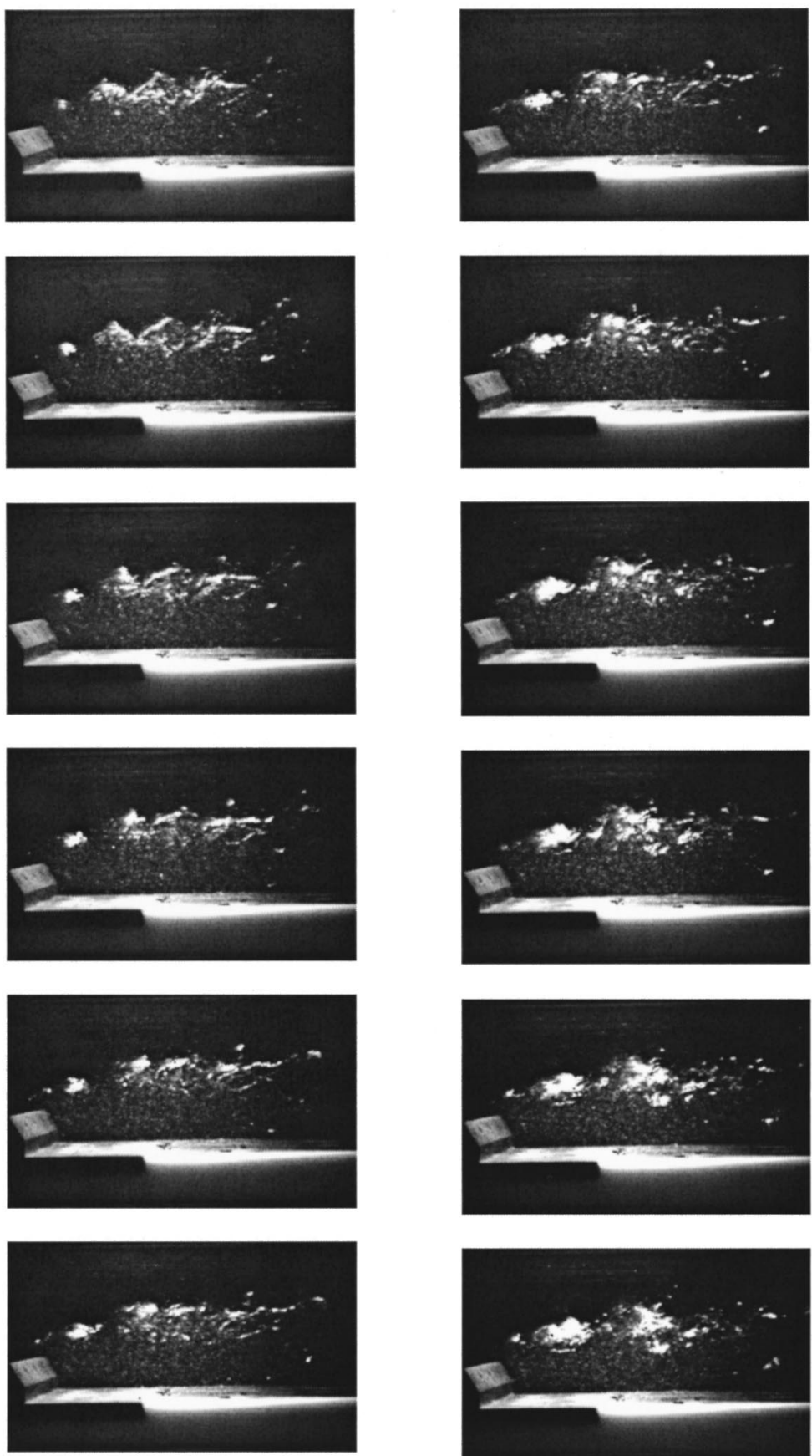
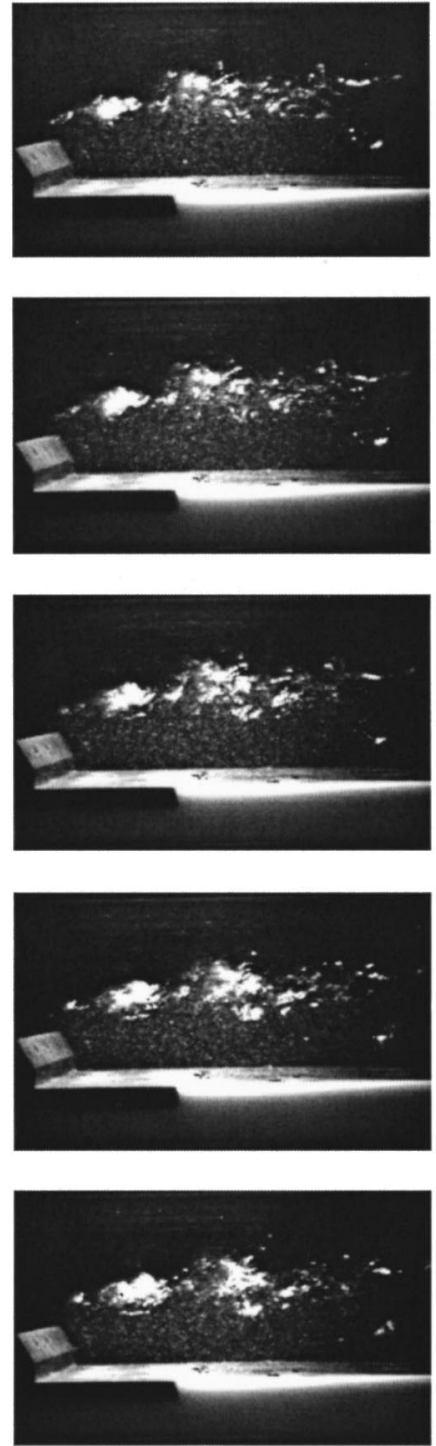
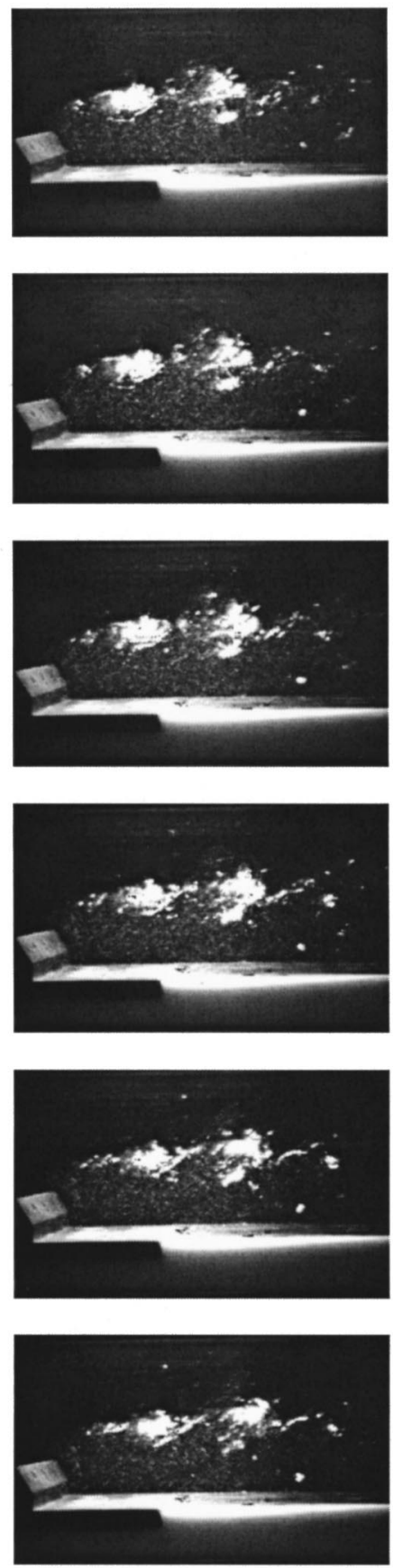

FIG. 6. A time series of images of shear-flow cavitation for $\sigma=0.52$ and $U_{\infty}=9.0 \mathrm{~m} / \mathrm{s}$. The flow is from left to right in each image. The series progresses from top to bottom and left to right. The time interval between each frame is $1.11 \mathrm{~ms}$.

where $\sigma_{i}$ is the cavitation number at inception. O'Hern ${ }^{17}$ examined cinemagraphic records of streamwise vortex cavitation inception, and the size of the initial bubble in the vortex at inception was determined. It was then assumed that the bubble radius was approximately equal to the original core radius, and this measurement was used to determine $\Gamma_{S}$. The spanwise vortex strength, $\Gamma_{P}$, was estimated to be $\Gamma_{P}$ $\approx \Delta U \cdot 1$, where $\Delta U$ is the velocity difference across the layer and 1 is the local spanwise vortex spacing. O'Hern concluded that the ratio $\Gamma_{P} / \Gamma_{s}$ was between 0.01 and 0.10 near the inception point of the streamwise vortices. This ratio was smaller than the value suggested by Jimenez et al. ${ }^{28}$ (who reported $\Gamma_{S} / \Gamma_{P} \approx 0.6 \pm 0.8$ for a single-phase plane shear layer). Recent investigations into developed tip-vortex cavitation suggest that incipient vortex cavitation does not fill the original viscous core (Briançon-Marjollet and $\left.\mathrm{Merle}^{29}\right)$. As the vortex fills with vapor, the diameter of the vapor tube may be several times the size of the original core size, but this is principally due to diffusion of gas into the vortex core. Arndt and Keller ${ }^{30}$ analyzed a Rankine vortex before and after the advent of developed cavitation. If angular momentum is conserved, the vapor core diameter will be $a / \sqrt{2}$. Examining the data at low air content $\left(\sigma_{i}=1.1\right)$, we will assume that the core size of noncavitating the vortex 


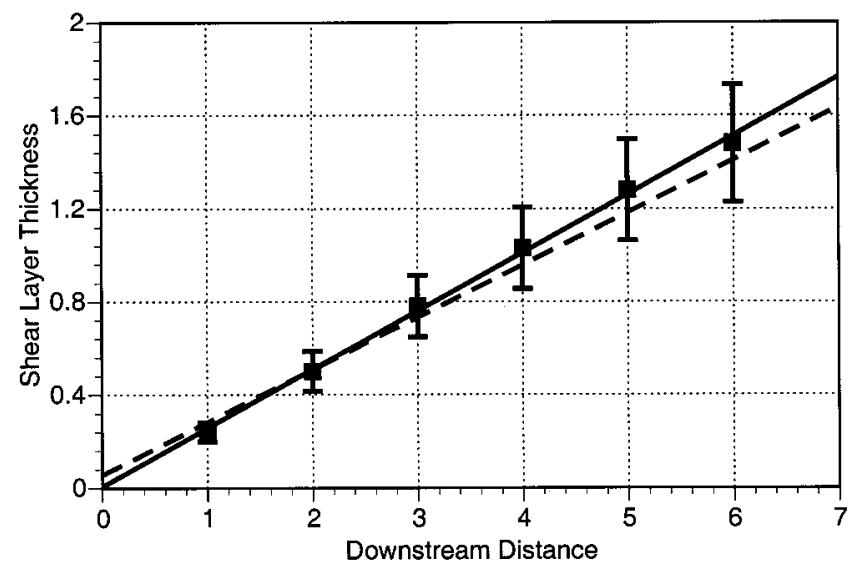

FIG. 7. Growth of shear layer thickness, $\delta^{*} / h$, as a function of downstream distance, $x / h$, for $\sigma=0.50$ and $U_{\infty}=9 \mathrm{~m} / \mathrm{s}$ (b). The solid line is the linear regression $\delta^{*} / h=0.25(x / h+0.02)$. These data are compared to that of O'Hern (1990), who found a relationship of $\delta^{*} / h=0.225(x / h+0.05)$ shown with the dashed line.

was 1.4 times the vapor core diameter. The average value of $\Gamma_{s}$ is then about $0.14 \mathrm{~m}^{2} / \mathrm{s}$, and $\Gamma_{P}$ is approximately $0.7 \mathrm{~m}^{2} / \mathrm{s}$. Then, $\Gamma_{S} / \Gamma_{P} \approx 0.2$. As we have assumed a larger initial core radius, this value is somewhat larger than that of O'Hern. However, it is still smaller than that expected from examination of noncavitating mixing layers.

\section{Void fraction of the cavitating shear layer}

Figure 10 presents a plot of the local void fraction measured in region A. Values reported are for two oxygen contents, and for both the light scattering and digital-imaging measurement methods. As the cavitation number decreases, the void fraction of the shear layer increases, as expected. However, the light scattering method consistently measured lower void fractions compared to the digital images measurement at higher cavitation numbers. The calculation of the void fraction from the bubble event rate employed a fixed bubble residence time, of $250 \mu \mathrm{s}$, in the measurement vol-

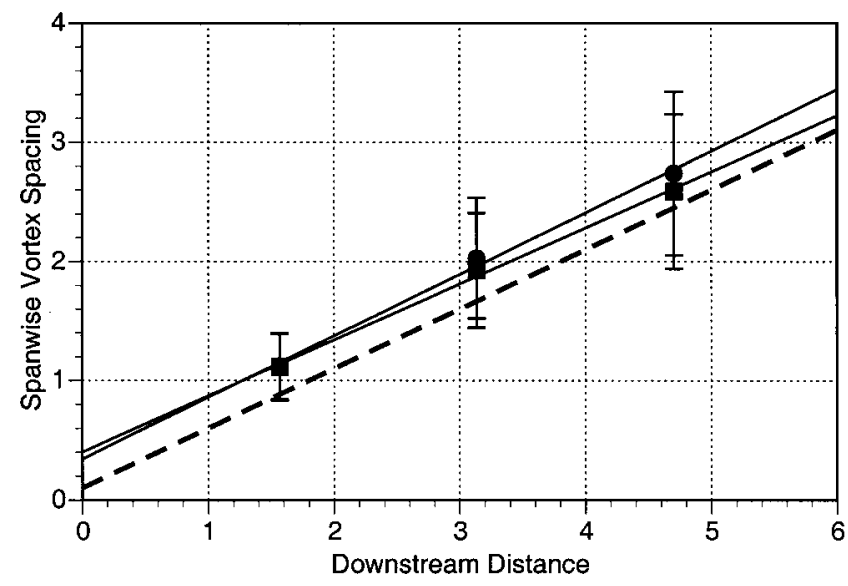

FIG. 8. Growth of the spanwise vortex spacing, $1 / h$, as a function of downstream distance, $x / h$, for $g=0.57(\bullet)$ and $\sigma=0.45(\mathbf{\square})$ and $U_{\infty}=9 \mathrm{~m} / \mathrm{s}$. The solid lines are linear regressions $1 / h=0.51(x / h+0.66)$ and $1 / h$ $=0.47(x / h+0.85)$. These data are compared to that of O'Hern (1990), who found a relationship of $1 / h=0.50(x / h+0.20)$ shown with a dashed line.

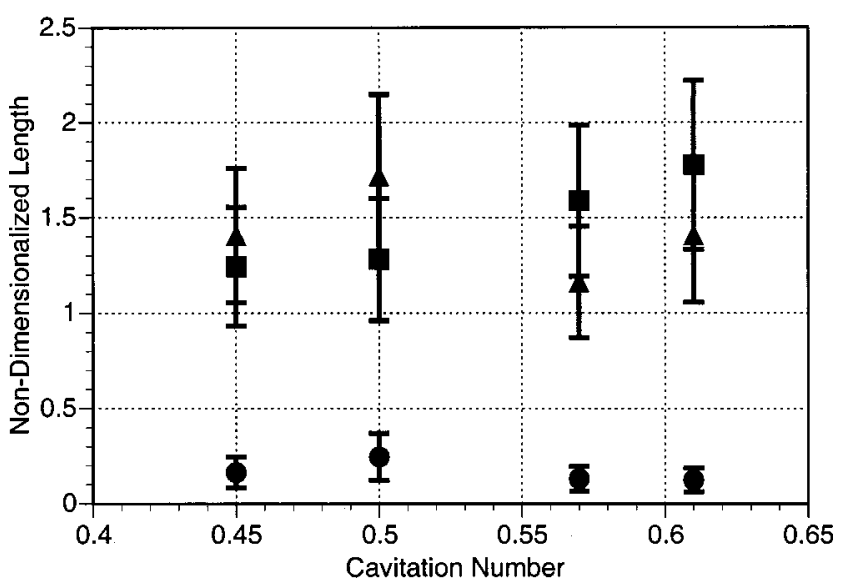

FIG. 9. The average spacing ( $\square$ ), length $(\mathbf{\Delta})$, and diameter $(\boldsymbol{O})$ of the streamwise vortices as a function of cavitation number observed in region A. The lengths are nondimensionalized by $2 \delta$.

ume. However, the actual residence time will likely increase with decreasing cavitation number as the average length of the streamwise bubbles increases. Moreover, the light scattering method measured the void fraction at a small volume within the center of the shear layer, while the light-sheet method averaged the void fraction over the entire region A. Yet, even with these differences, the void fractions are qualitatively similar with maximum average void fractions of $1 \%-1.5 \%$ at the lowest cavitation numbers. Note that the average void fraction is reduced as the dissolved oxygen content is reduced for a given cavitation number. A reduction in dissolved gas content is often associated with a reduction in the free gas content (i.e., the number of small free-stream bubbles), and with a reduction in the number of free-stream nuclei the extent of the cavitation will be reduced.

\section{Mean pressure}

The mean pressure difference measured between the surface taps T1 and T2, shown in Fig. 1, was 5.8 $\pm 0.2 \mathrm{kPa}$. This

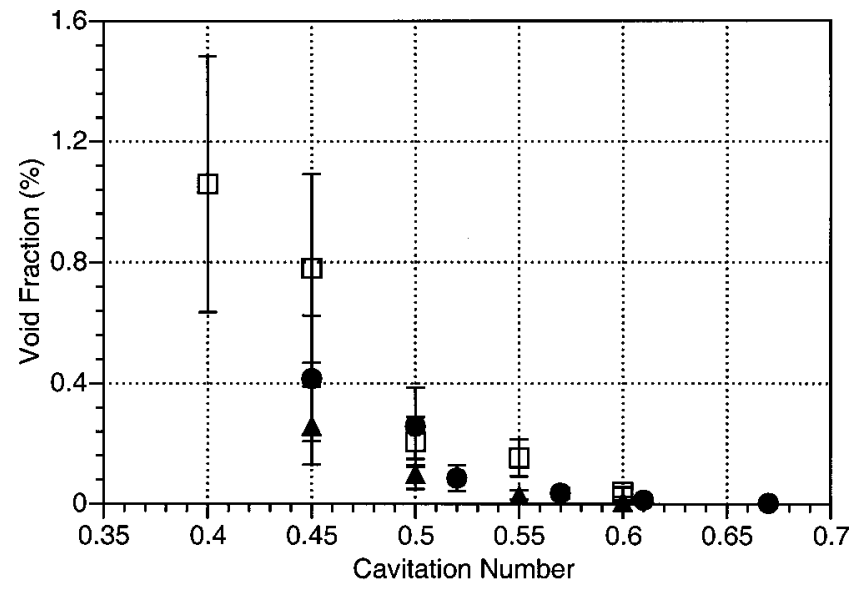

FIG. 10. Void fraction in region $\mathrm{A}$ as a function of cavitation number. Two methods were used to determine the void fraction. Light scattering was employed at a single point in the center of region A for $3 \mathrm{ppm}(\mathbf{)})$ and 1 ppm ( $\mathbf{\Delta})$ dissolved oxygen content. The void fraction was inferred from planar digital images in region A for 3 ppm dissolved oxygen content ( $\square$ ). 

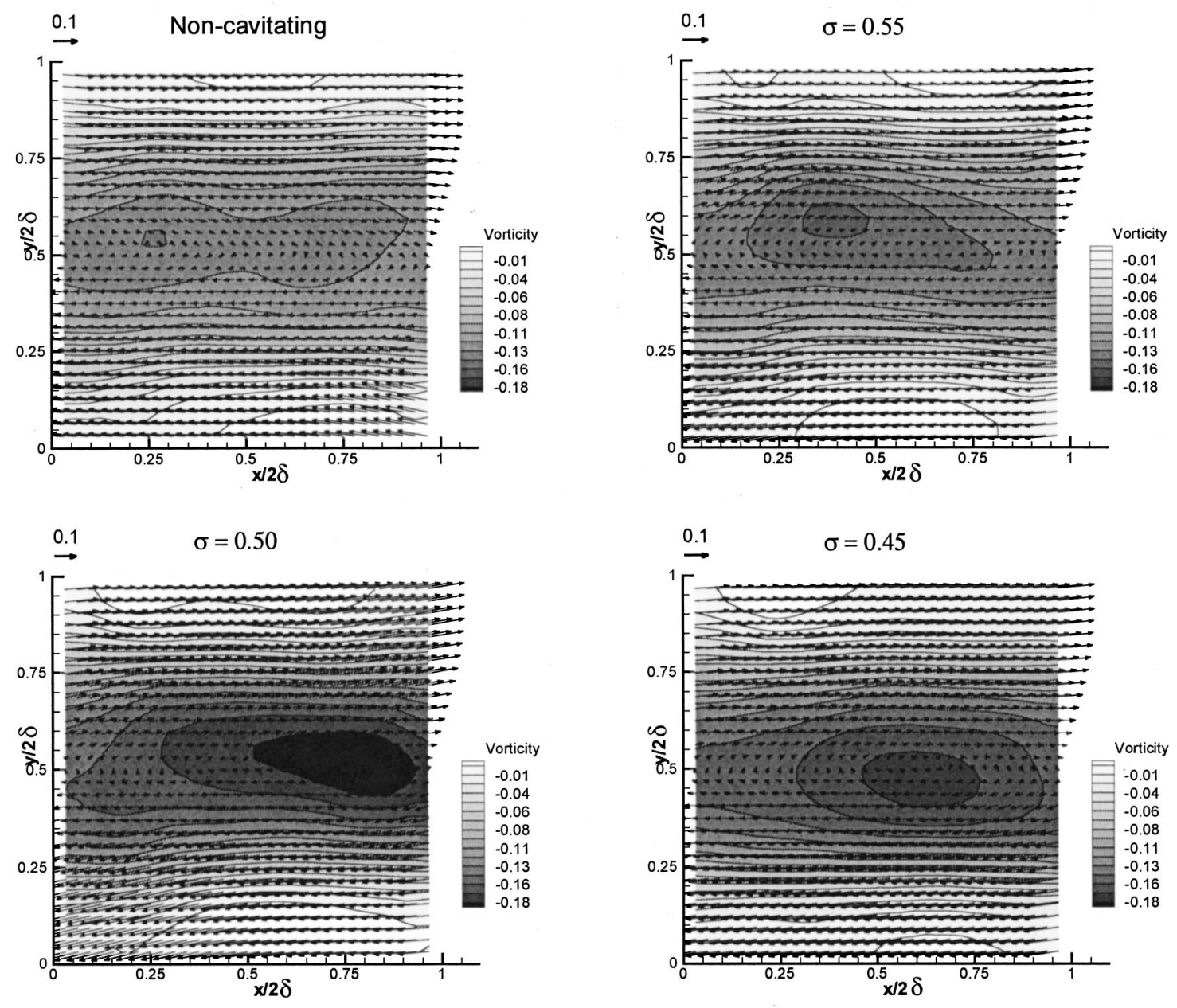

FIG. 11. Average flow in region B presenting the nondimensional mean velocity, $\left(\bar{u}-U_{\text {Mean, } \mathrm{B}}\right) / U_{\text {Mean, } \mathrm{B}}$ and the nondimensional vorticity contours, $\omega_{z} 2 \delta / U_{\text {Mean,B }}$. The flow is from left to right. Here 1000 individual images were averaged.

value was not influenced by the extent of cavitation within the test section over the range of cavitation numbers examined. This suggests that the mean flow through the test section was not substantially altered by the development of cavitation in the shear layer.

\section{E. Comparison between the noncavitating and the cavitating mean flows in region $B$}

As the shear layer enters the diffuser, the vapor pockets in the cavitating vortices collapse leaving only small gas bubbles in the flow. PIV measurements were performed in this downstream region of the cavitating flow using only seed particles as flow tracers. Measurements were performed downstream of the noncavitating shear flow $(\sigma=2.3)$ and downstream of three cavitating shear flows, with cavitation numbers of 0.55, 0.50, and 0.45. As shown in Fig. 10, the void fraction of the cavitating shear layer increases sharply between $\sigma=0.50$ and 0.45 . By conducting measurements in region $\mathrm{B}$, the lasting influences of the growth and collapse of the cavitating vortices on the turbulent shear flow are examined.

One-thousand PIV images were acquired in region B and averaged for each cavitation number. Figure 11 presents the dimensionless mean in-plane velocity, $(\bar{u}$ $\left.-U_{\text {Mean, } \mathrm{B}}\right) / U_{\text {Mean, } \mathrm{B}}$, superimposed on the dimensionless outof-plane vorticity, $\omega_{z} 2 \delta / U_{\text {Mean }, \mathrm{B}}$. The mean velocity, $U_{\text {Mean, } \mathrm{B}}$, has been subtracted from each image. Some differences are observed in the mean velocity field of the noncavitating flow compared to the cavitating shear flow. Figure 12 shows the average velocity profiles, $\bar{u} / U_{\mathrm{fs}}$, for three crossstream sections of region B: $x / h=20.54$ in the left half (upstream) of the image, $x / h=20.96$ in the middle of the image, and $x / h=21.38$ in the right half (downstream) of the image. The average free-stream velocity, $U_{\mathrm{fs}}$, was computed by averaging 25 velocity vectors from the top left corner of the average velocity field.

At the lowest cavitation numbers, the gradient of the velocity across region $B$ is increased. The influence of the cavitation decreases with increasing downstream distance. Convection of the flow across region B represents nearly one turnover of the spanwise eddies. Figures 13 and 14 present profiles of the average vorticity and strain rate for the same three cross-streams. These data are normalized by $2 \delta$, the average shear layer thickness of region A, since for the purpose of scaling $2 \delta$ is the order of magnitude of the shear layer thickness in region B. With $\Delta u / U_{\mathrm{fs}} \sim \pm 0.02$, and with 


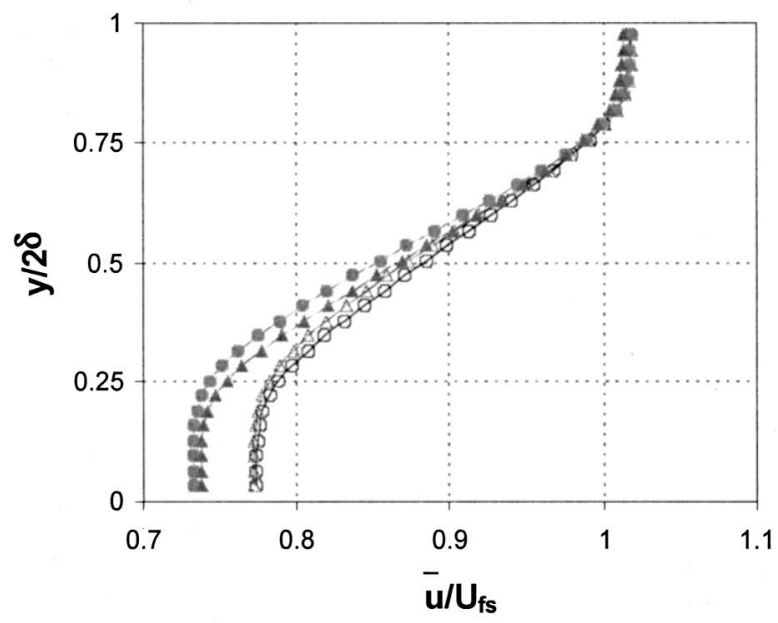

(a)

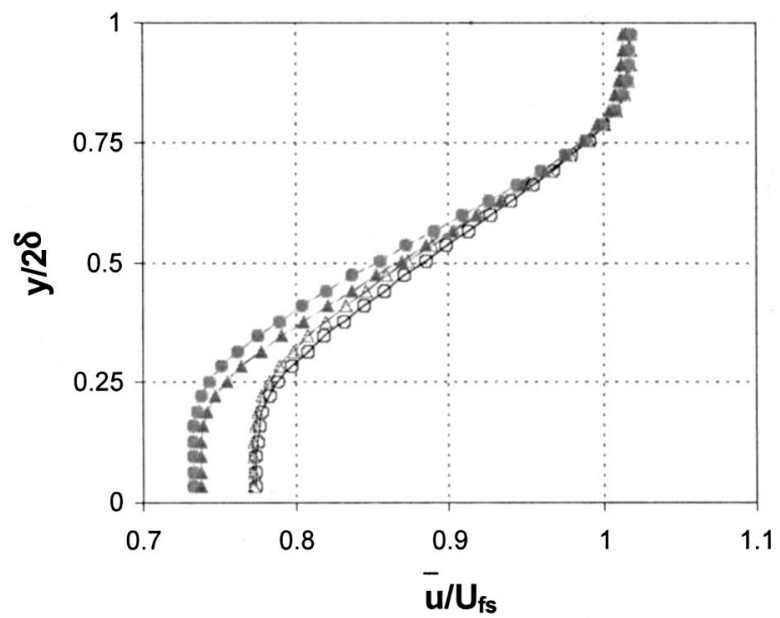

(b)

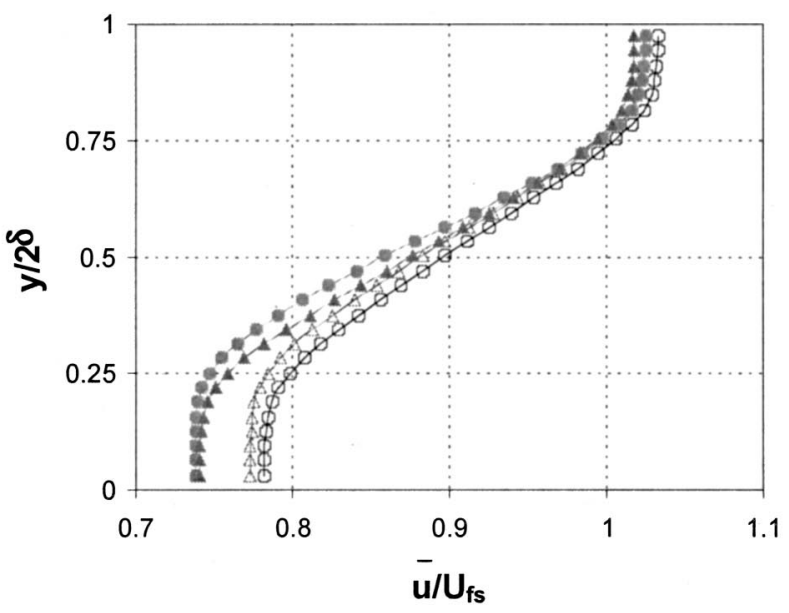

(c)

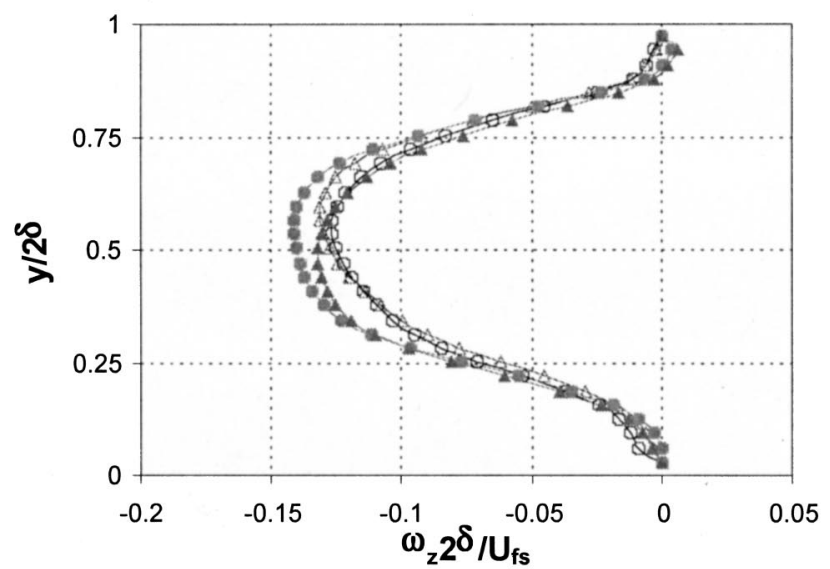

(a)

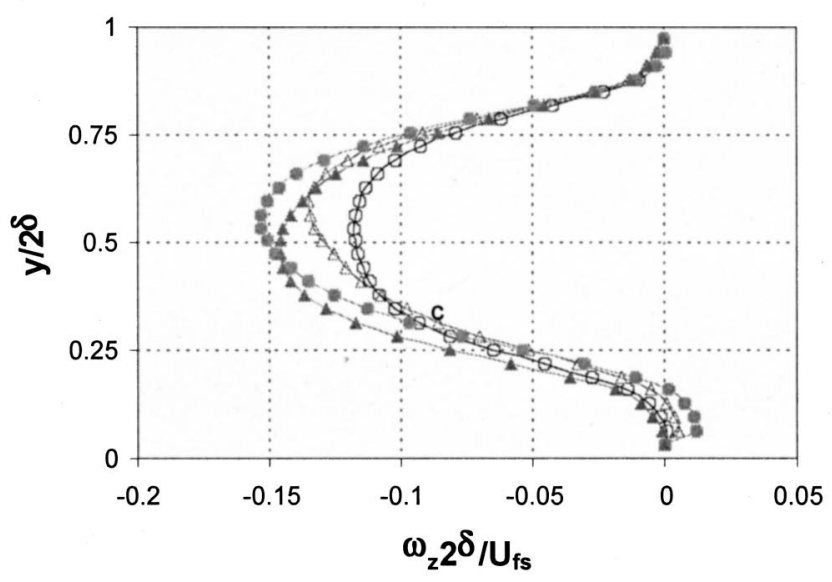

(b)

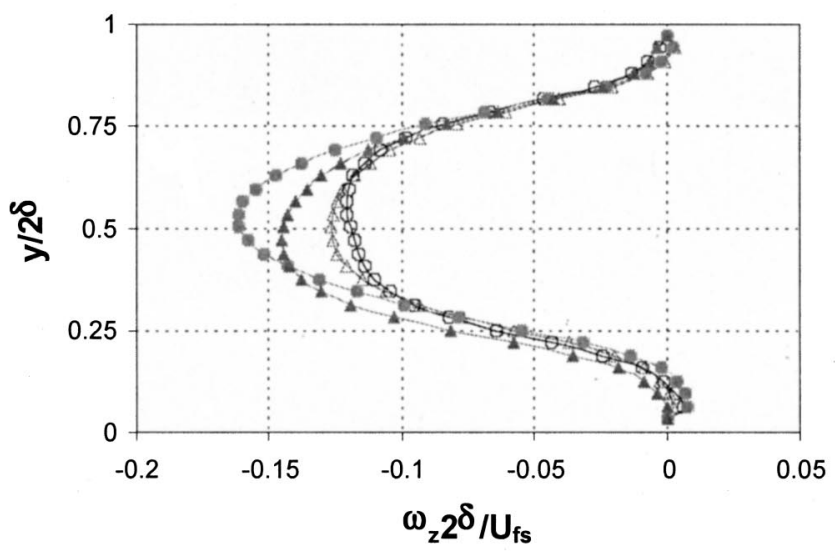

(c)

FIG. 13. Average vorticity profiles, $\omega_{z} 2 \delta / U_{\mathrm{fs}}$, as a function of cavitation number for three cross-stream sections of region $\mathrm{B}$ : (a) $x / h=20.54$, (b) $x / h=20.96$, (c) $x / h=21.38$. $\bigcirc$ is the noncavitating case, $\Delta$ is $\sigma=0.55$, $\bigcirc$ is $\sigma=0.50$, and $\boldsymbol{\Delta}$ is $\sigma=0.45$.

an uncertainty in the position of $\Delta x / 2 \delta \sim 0.001$, the uncertainty in the nondimensionalized vorticity and strain rate is approximately \pm 0.10 . After averaging, the uncertainty in the vorticity and strain profiles is \pm 0.003 . Differences of less than \pm 0.006 are within the uncertainty of the measurement.
FIG. 12. Average velocity profiles, $u / U_{\mathrm{fs}}$, as a function of cavitation number for three cross-stream sections of region B derived from the data in Fig. 13. (a) $x / h=20.54$, (b) $x / h=20.96$, (c) $x / h=21.38$. The symbols $\bigcirc$ is for the noncavitating case, $\Delta$ is $\sigma=0.55,0$ is $\sigma=0.50$, and $\boldsymbol{\Delta}$ is $\sigma=0.45$. Here 1000 individual PIV images were averaged. 


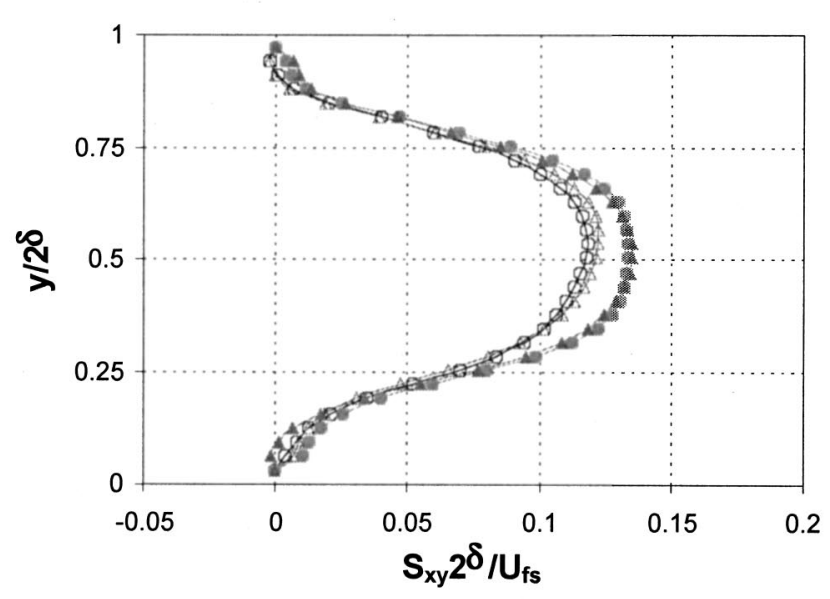

(a)

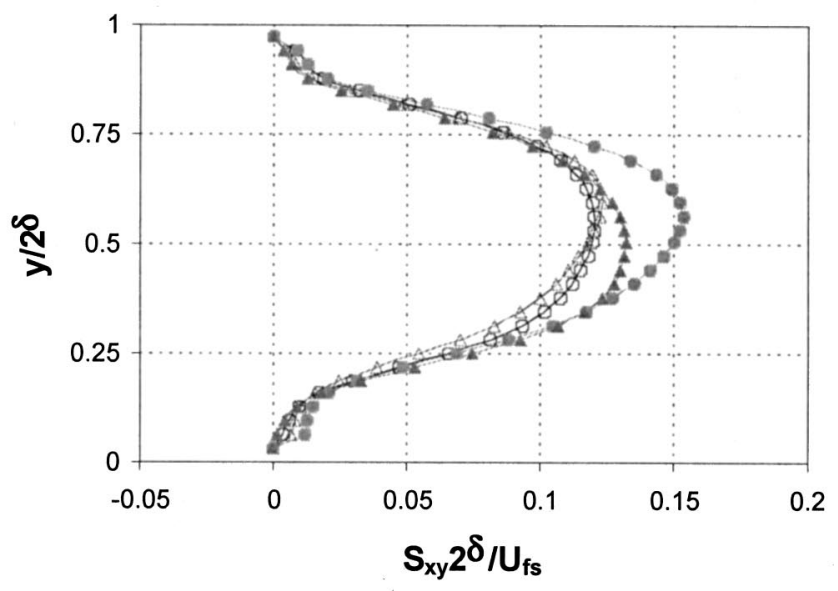

(b)

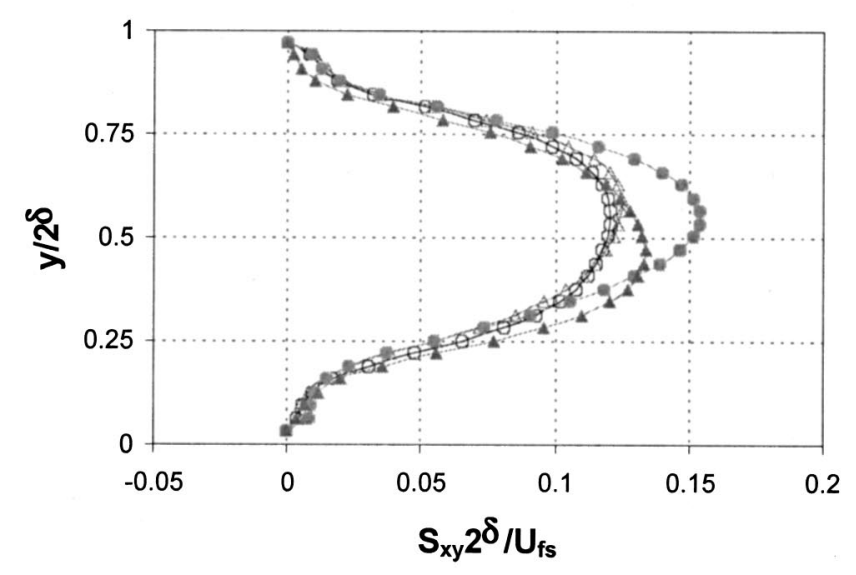

(c)

FIG. 14. Average strain rate profiles, $S_{x y} 2 \delta / U_{\mathrm{fs}}$, as a function of cavitation number for three cross-stream sections of region B: (a) $x / h=20.54$, (b) $x / h=20.96$, (c) $x / h=21.38$. $\bigcirc$ is the noncavitating case, $\triangle$ is $\sigma=0.55$, $\square$ is $\sigma=0.50$, and $\boldsymbol{\Delta}$ is $\sigma=0.45$.

The maximum absolute vorticity levels are higher in the cavitating flows than in the noncavitating flow, especially for the cavitation numbers $\sigma=0.50$ and $\sigma=0.45$. Similar results for the strain rate profiles are shown in Fig. 14. The higher vorticity and strain rates are consistent with the decrease in the shear layer thickness at lower cavitation numbers. The case of $\sigma=0.50$ is again anomalous with the highest maximum vorticity and strain rate magnitudes. A possible explanation for this may be the significant increase in the void fraction beneath the shear layer at the lowest cavitation numbers. This is observed in Figs. 5(d) and 5(e). Increasing concentration of bubbles beneath the shear layer reduces the mean density of this recirculating flow. This, in turn, would reduce the shear stresses across the layer upstream of region B.

\section{F. Comparison between the turbulent fluctuations of the noncavitating and the cavitating flows in region $B$}

Figures 15, 16, and 17 present profiles of the mean Reynolds stresses $\overline{u^{\prime} u^{\prime}} / U_{\mathrm{fs}}^{2}, \overline{v^{\prime} v^{\prime}} / U_{\mathrm{fs}}^{2}$, and $-\overline{u^{\prime} v^{\prime}} / U_{\mathrm{fs}}^{2}$ at the three cross-stream locations. The average velocity at each vector location was determined, and this local average velocity was subtracted from each vector to determine the fluctuation velocity components $u^{\prime}$ and $v^{\prime}$ for each image. This data was then used to compute the average Reynolds stresses. Figure 18 shows the convergence plots for the Reynolds stresses at a point in the middle of the domain. It indicates that 1000 images are sufficient to achieve a statistically steady average.

Consider the trends in $\overline{u^{\prime} u^{\prime}} / U_{\mathrm{fs}}^{2}$ in Fig. 15. First, it should be noted that the location of maximum fluctuations is not in the center of the shear layer. By the time the flow is in region $\mathrm{B}$, the shear layer is in the diffuser and is no longer similar to the planar mixing layer of region A. The $\overline{u^{\prime} u^{\prime}} / U_{\mathrm{fs}}^{2}$ profiles are not significantly changed with decreasing cavitation number, and the magnitude of the fluctuations increases with the extent of cavitation upstream. Laberteaux and Ceccio ${ }^{5}$ showed that the collapse of cavitation bubbles leads to the production of turbulence within the flow, and this increase in streamwise turbulence may result from this mechanism. Again, the case of $\sigma=0.50$ is anomalous, as the level of turbulence observed at the lower boundary of the shear layer is reduced for just this case.

For the measurements of $\overline{v^{\prime} v^{\prime}} / U_{\mathrm{fs}}^{2}$ (Fig. 16), the presence of cavitation has reduced the extent of the maximum cross-stream fluctuations compared with the noncavitating flow by as much as $30 \%$. These results suggest that the presence of cavitation either damps out the cross-stream fluctuations or reduces their production. Similar trends are found in the measurements of $-\overline{u^{\prime} v^{\prime}} / U_{\mathrm{fs}}^{2}$ (Fig. 17), where an increase in the extent of cavitation reduced the correlation between the streamwise and cross-stream velocities. The streamwise stretching of a vortex will lead to an increase in the rotation rate of the vortex; thus, streamwise velocity fluctuations are coupled to cross-stream fluctuations. Belahadji et al. ${ }^{22}$ suggested that the presence of vapor in the cores of such vortices modifies the vortex-stretching process by decoupling the rate of vortex straining from the rotation rate. As a noncavitating vortex is stretched, the conservation of angular momentum implies that the rotation rate will increase and the pressure in the viscous core will decrease. However, the core of a cavitating vortex can maintain a constant pressure, and stretching 


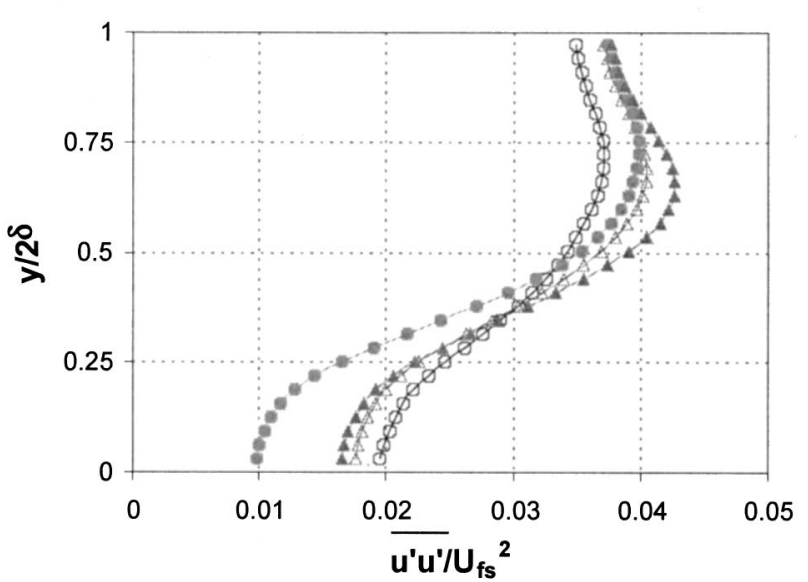

(a)

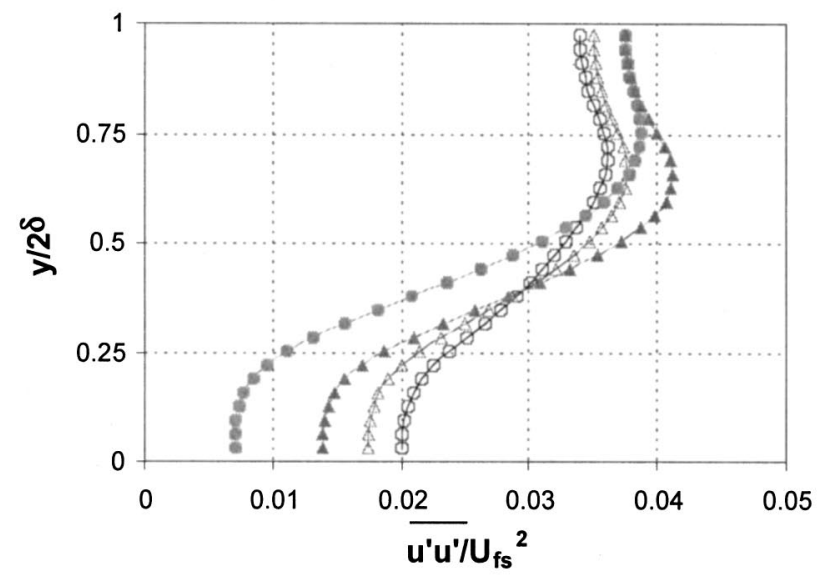

(b)

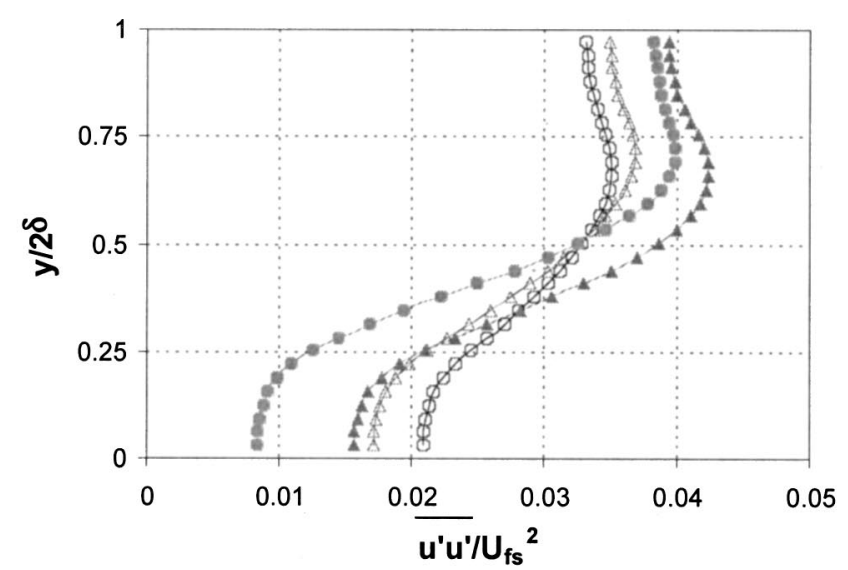

(c)

FIG. 15. Mean Reynolds stress profiles, $\overline{u^{\prime} u^{\prime}} / U_{\mathrm{fs}}^{2}$, as a function of cavitation number for three cross-stream sections of region B: (a) $x / h=20.54$, (b) $x / h=20.96$, (c) $x / h=21.38$. $\bigcirc$ is the noncavitating case, $\triangle$ is $\sigma=0.55$, $\bigcirc$ is $\sigma=0.50$, and $\boldsymbol{\Delta}$ is $\sigma=0.45$.

will result only in the production of more core vapor with little change in the vortex core diameter. Such a mechanism could be responsible for a decrease in the production of cross-stream fluctuations and a reduction in the stress $-\overline{u^{\prime} v^{\prime}} / U_{\mathrm{fs}}^{2}$, as seen in the data. However, these distributions

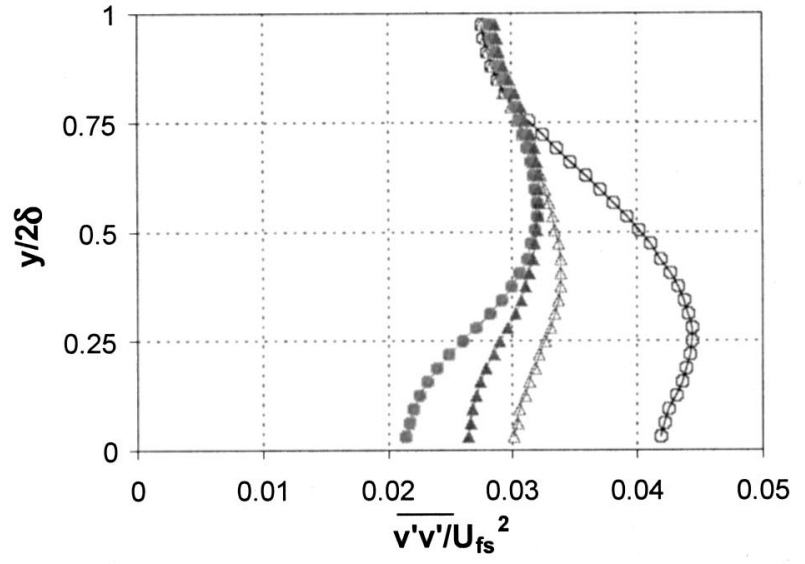

(a)

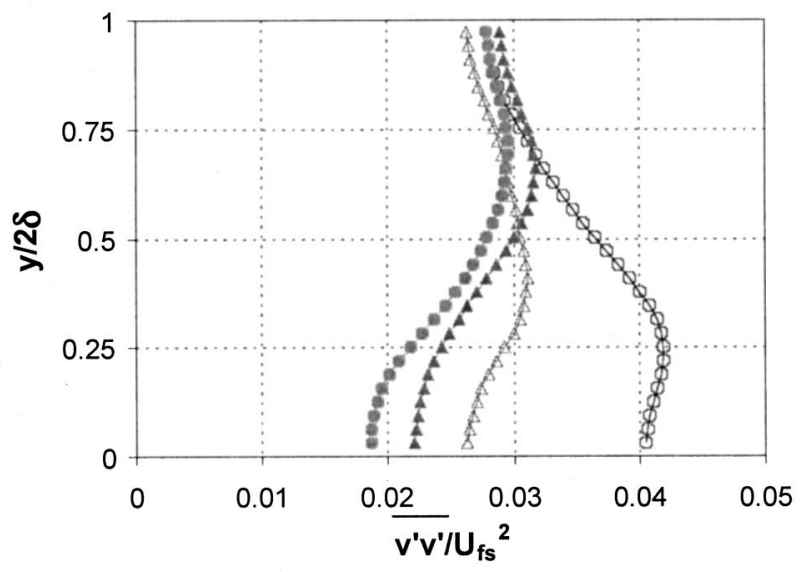

(b)

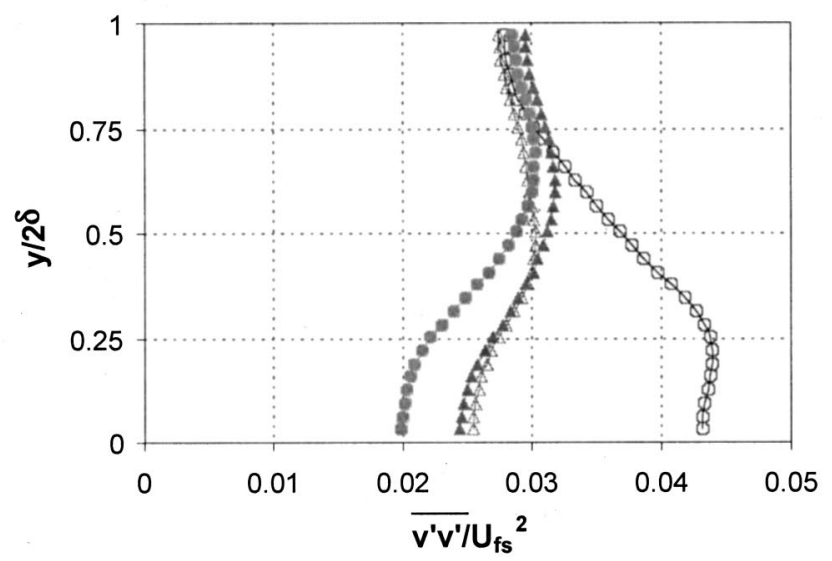

(c)

FIG. 16. Mean Reynolds stress profiles, $\overline{v^{\prime} v^{\prime}} / U_{\mathrm{fs}}^{2}$, as a function of cavitation number for three cross-stream sections of region B: (a) $x / h=20.54$, (b) $x / h=20.96$, (c) $x / h=21.38$. $\bigcirc$ is the non-cavitating case, $\Delta$ is $\sigma=0.55$, is $\sigma=0.50$, and $\boldsymbol{\Delta}$ is $\sigma=0.45$.

also exhibit some anomalies. Once more, the case of $\sigma$ $=0.50$ shows the largest reductions in average Reynolds stresses compared to the noncavitating case. And, the case of $\sigma=0.45$ shows increasing values of $-\overline{u^{\prime} v^{\prime}} / U_{\mathrm{fs}}^{2}$ near the top edge of the shear layer. 


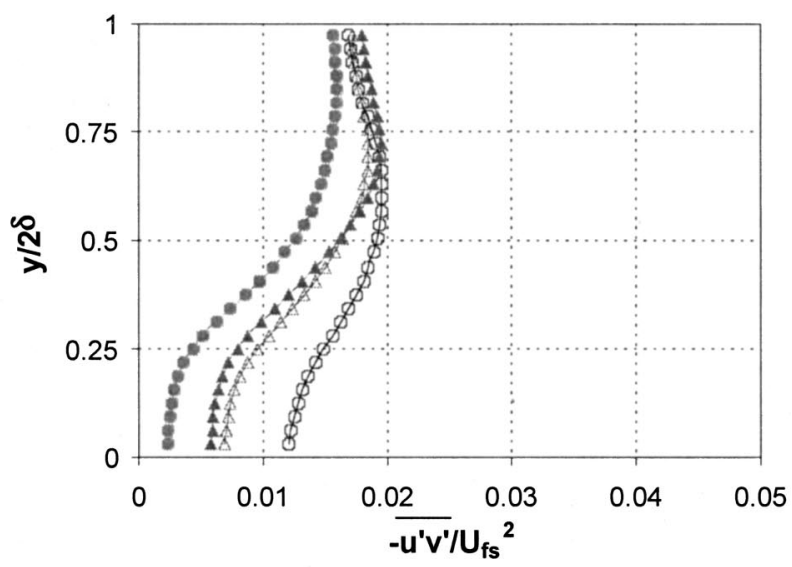

(a)

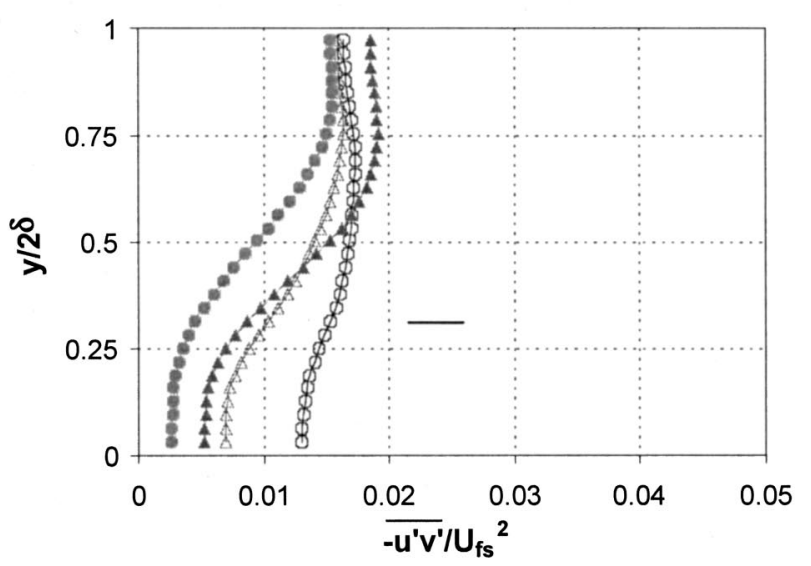

(b)

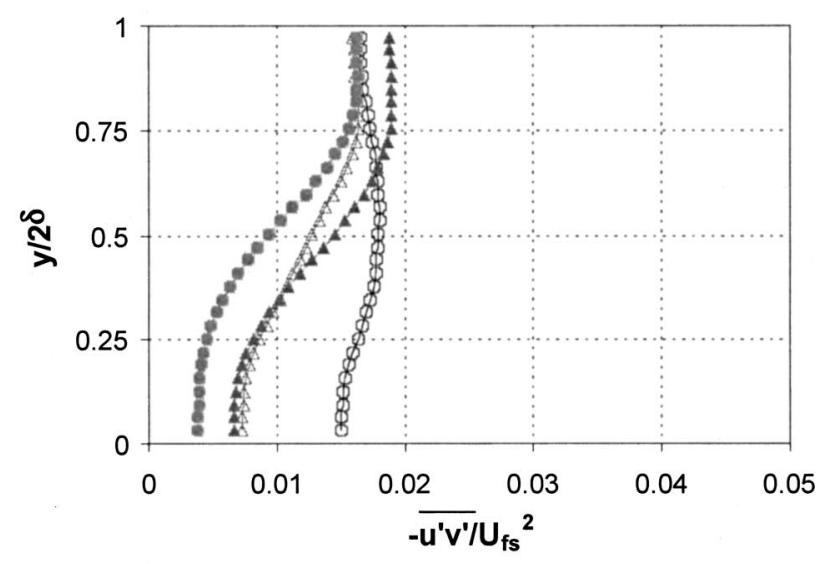

(c)

FIG. 17. Mean Reynolds stress profiles, $-\overline{u^{\prime} v^{\prime}} / U_{\mathrm{fs}}^{2}$, as a function of cavitation number for three cross-stream sections of region B: (a) $x / h=20.54$, (b) $x / h=20.96$, (c) $x / h=21.38 . \bigcirc$ is the non-cavitating case, $\Delta$ is $\sigma$ $=0.55$, is $\sigma=0.50$, and $\boldsymbol{\Delta}$ is $\sigma=0.45$.

The PIV images were analyzed to reveal the probability distributions of the instantaneous vorticity and strain rates measured within region B. Figures 19 and 20 show the probability distribution for the values of vorticity and strain rate taken from 1000 images. Differences are only observed at

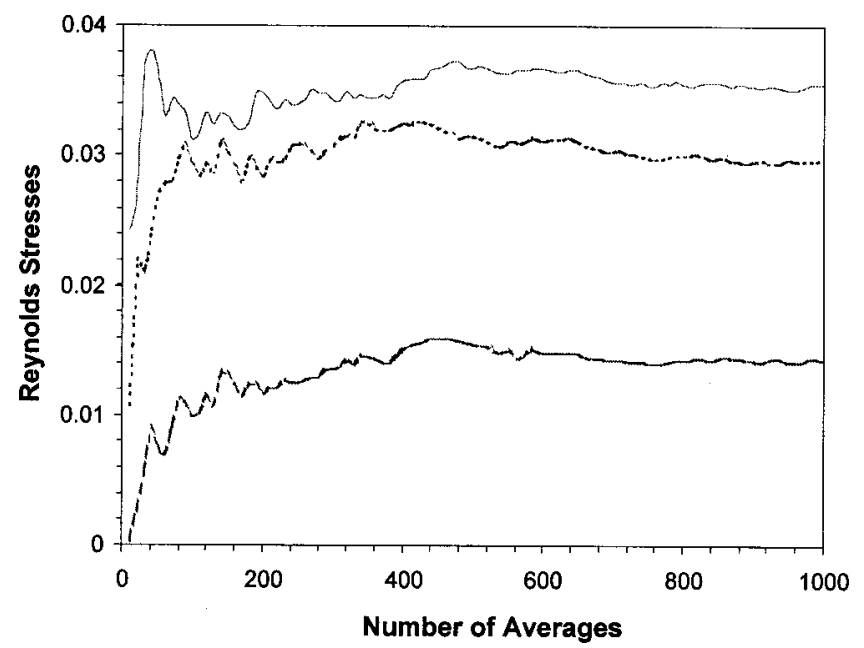

FIG. 18. Convergence plot for the mean Reynolds stresses at a point in the middle of region $\mathrm{B}: \overline{u^{\prime} u^{\prime}} / U_{\mathrm{fs}}^{2}$ (solid line); $\overline{v^{\prime} v^{\prime}} / U_{\mathrm{fs}}^{2}$ (short-dashed line); and $-\overline{u^{\prime} v^{\prime}} / U_{\mathrm{fs}}^{2}$ (long-dashed line).

the extreme positive and negative values, and the trends with cavitation number observed in the profiles can be detected in variations of the histograms. For the case of $\sigma=0.50$, there is a slight shift toward a higher probability of strong negative vorticity and strong positive strain rates, and these trends reverse for the condition of $\sigma=0.45$.

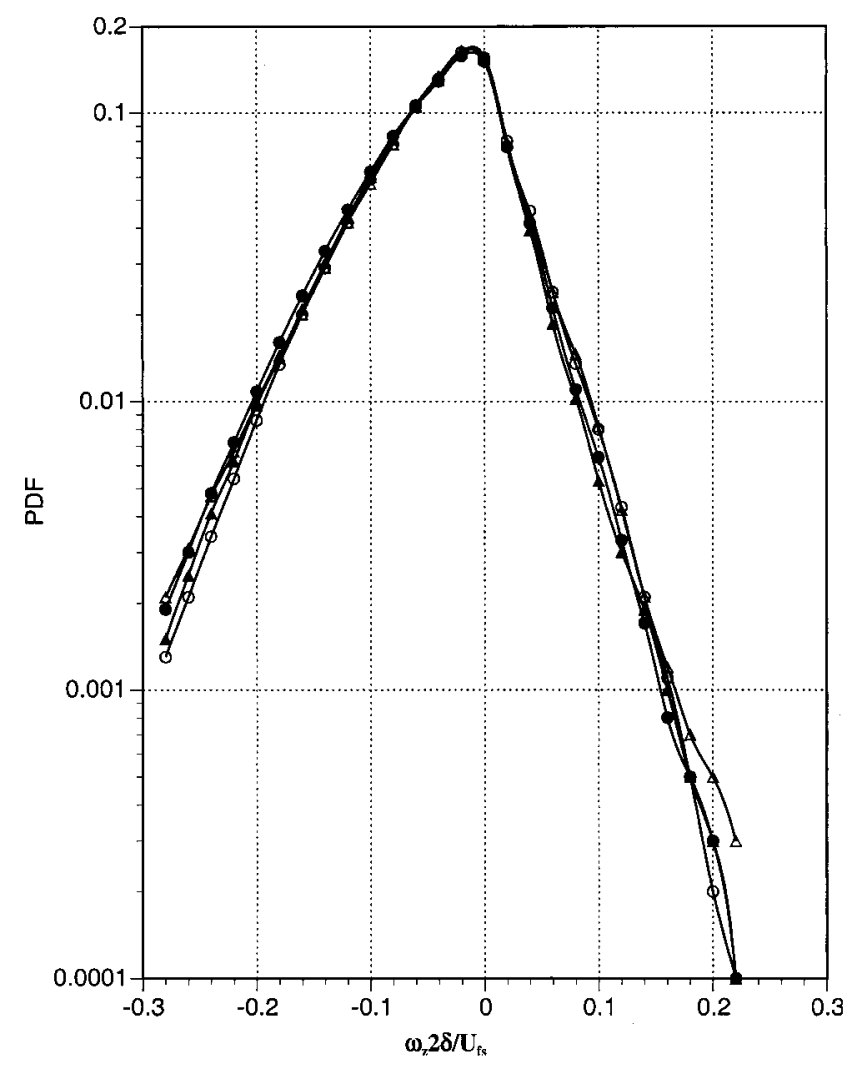

FIG. 19. Probability density function of the normalized vorticity, $\omega_{z} 2 \delta / U_{\mathrm{fs}}$, measured in region $\mathrm{B}$ for varying cavitation numbers. The measurements from 1000 PIV images are presented. $\bigcirc$ is the noncavitating case, $\triangle$ is $\sigma$ $=0.55,0$ is $\sigma=0.50$, and $\mathbf{\Delta}$ is $\sigma=0.45$. 


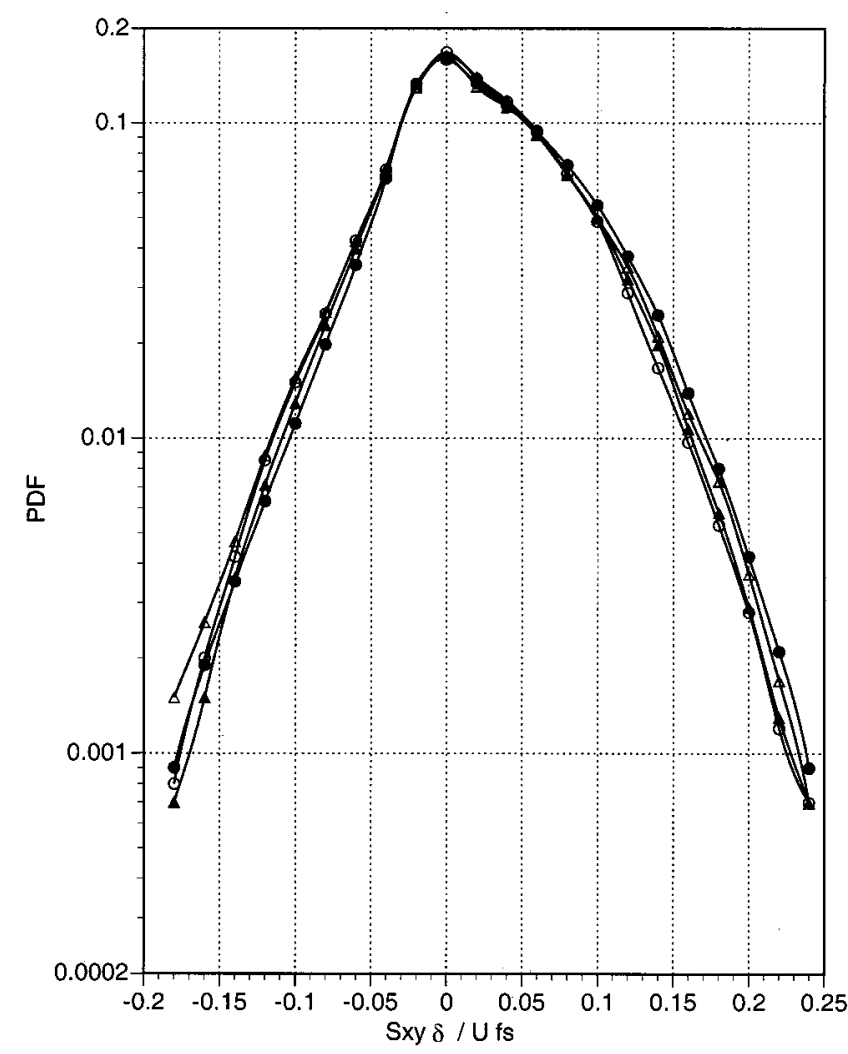

FIG. 20. Probability density function of the normalized strain rate, $S_{x y} \delta / U_{\mathrm{fs}}$, measured in region B for varying cavitation numbers. The measurements from 1000 PIV images are presented. $\bigcirc$ is the noncavitating case, $\triangle$ is $\sigma=0.55, \bigcirc$ is $\sigma=0.50$, and $\boldsymbol{\Delta}$ is $\sigma=0.45$.

\section{G. Influence of cavitation on the shear stress across the shear layer}

The data presented above indicate that the shear layer was modified by the cavitation, but only subtly. As we are examining the flow after the collapse of the cavitation bubbles, interpretation of the results can be problematic, but it appears that the cavitation resulted in an increase of the mean shear rate across the layer and a decrease in the Reynolds stresses within the layer. While the flow examined here is not a classical mixing layer, the scaling of a variable density mixing layer may be useful in examining this flow (see Hermanson and Dimotakis ${ }^{31}$ for a complete derivation). A similarity variable can be defined as

$$
\eta=y / \delta(x),
$$

where $x$ is the streamwise direction and $y$ is the cross-stream direction. The velocity distribution across the shear layer takes the form

$$
U(\eta)=\hat{s} U_{1} \frac{d f(\eta)}{d \eta}
$$

and

$$
\widetilde{V}(\eta)=\hat{s} U_{1}\left(\eta \cdot \frac{d f(\eta)}{d \eta}-f(\eta)\right) \frac{d \delta}{d x},
$$

where $U$ is the mean streamwise velocity, $\rho \widetilde{V}=\rho V+\overline{\rho^{\prime} v^{\prime}}$, and $U_{1}$ is the velocity of the high-speed stream on the mix- ing layer. The density ratio $\hat{s}(\eta)=\rho / \rho_{S}$ compares the freestream density to the local shear layer density. Substitution into the streamwise momentum equation yields

$$
-\frac{U_{1}^{2}}{\Delta U^{2}} \frac{d \delta}{d x} f\left(\frac{d \hat{s}}{d \eta} f+\hat{s} \frac{d^{2} f}{d \eta^{2}}\right)=\frac{1}{\rho \Delta U^{2}} \frac{d \tau}{d \eta}-\frac{\eta}{\rho \Delta U^{2}} \frac{d P}{d \eta} \frac{d \delta}{d x},
$$

where $\tau=-\rho \overline{u^{\prime} v^{\prime}}$ is the Reynolds shear stress plus higherorder terms and $\Delta U$ is the velocity difference across the shear layer. Here, it is assumed that streamwise velocity fluctuation correlations are small compared to $U^{2}$, and that the Reynolds stresses are much larger than the viscous stresses. The pressure gradient term on the right-hand side is small compared to the shear-stress term. The second term on the left results from density gradients in the shear layer. The effect of buoyancy has been neglected here as the flow is momentum dominated. The Richardson number is

$$
\mathrm{Ri} \approx \frac{\alpha g \delta_{0}}{U_{\mathrm{fs}}^{2}} \approx 10^{-5},
$$

where $g$ is the gravitational constant.

While the advent of cavitation alters the local density of the shear layer, the maximum reduction in the mean density within the shear layer is less than $1 \%$, based on the void fraction measurements. This is in contrast to reacting shear layers, where the density reduction variation can be as high as $60 \%$. We will assume here that the gradient of density across the shear layer is small and can be neglected. The streamwise momentum equation becomes

$$
-\hat{s} \frac{U_{1}^{2}}{\Delta U^{2}} \frac{d \delta}{d x} \frac{d^{2} f}{d \eta^{2}} f \approx \frac{1}{\rho \Delta U^{2}} \frac{d \tau}{d \eta} .
$$

This equation can be integrated numerically for a given measured velocity distribution with the constraint that the shear stresses vanish in the two free-streams. A constraint based on mass conservation can be applied to achieve this result (Hermanson and Dimotakis ${ }^{31}$ ).

Figure 21 shows the velocity profiles measured in region B [from Fig. 12(a)] scaled with the measured velocity difference across the layer. The profiles are nearly identical, and they are approximated by the function $\tanh [2(y / \delta-1)]$, and this is approximately the form of

$$
\frac{d f(\eta)}{d \eta}=\frac{\Delta U}{2 U_{1}} \tanh [2(y / \delta-1)] .
$$

The function $f(\eta)$ can be found by integrating $d f(\eta) / d \eta$. The maximum value of the shear stress is found on the dividing streamline, where $\widetilde{V}=\eta_{0} U$. Let us assume that the profiles across the layer are symmetric in $\left(\eta-\eta_{0}\right)=\hat{\eta}$. Both the shear-stress distribution $\tau(\hat{\eta})$ and $d^{2} f(\hat{\eta}) / d \hat{\eta}^{2}$ are even functions of $\hat{\eta}$. Consequently, $f(\hat{\eta})$ is an odd function of $\hat{\eta}$. An approximate expression for $f(\hat{\eta})$ is

$$
f(\hat{\eta}) \approx \frac{\Delta U}{U_{1}} \hat{\eta} .
$$

Also, the derivative of $d f(\hat{\eta}) / d \hat{\eta}$ yields 


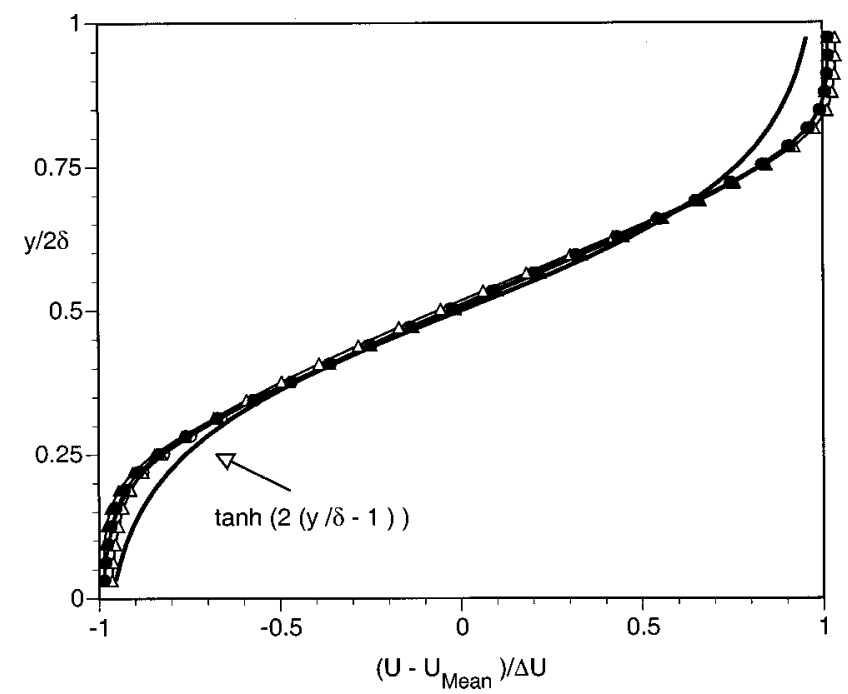

FIG. 21. Scaled velocity profiles, $\left(\bar{u}-U_{\text {Mean }}\right) / \Delta U$, as a function of cavitation number for three cross-stream sections of region B for $x / h=20.54$ derived from the data in Fig. 14(a): $\bigcirc$ is the noncavitating case, $\triangle$ is $\sigma$ $=0.55, \boldsymbol{O}$ is $\sigma=0.50$, and $\boldsymbol{\Delta}$ is $\sigma=0.45$. Also shown is the velocity profile $\tanh [2(y / \delta-1)]$.

$$
\frac{d^{2} f(\hat{\eta})}{d \hat{\eta}^{2}} \approx \frac{\Delta U}{U_{1}} \operatorname{sech}^{2}(2 \hat{\eta})
$$

These expressions can be integrated to determine an expression for the shear stress across the shear layer:

$$
\int f \frac{d^{2} f}{d \hat{\eta}^{2}} d \hat{\eta} \approx \frac{\Delta U}{2 U_{1}}[2 \hat{\eta} \tanh (2 \hat{\eta})-\ln \cosh (2 \hat{\eta})-C],
$$

where $C$ is a constant chosen such that the shear stress tend to zero for large magnitudes of $\hat{\eta}$. In the limit as $\hat{\eta} \rightarrow \infty$, $2 \hat{\eta} \tanh (2 \hat{\eta})-\ln \cosh (2 \hat{\eta}) \rightarrow \ln (2)=0.693 \approx C$. The shear stress distribution is then given by

$$
-\frac{1}{4} \frac{d \delta}{d x}[2 \hat{\eta} \tanh (2 \hat{\eta})-\ln \cosh (2 \hat{\eta})-C] \approx \frac{\tau}{\rho \Delta U^{2}} .
$$

The maximum shear stress at $\hat{\eta}=0$ is then

$$
C \frac{1}{4} \frac{d \delta}{d x} \approx \frac{\tau_{\max }}{\rho \Delta U^{2}} .
$$

This scaling indicates that the magnitude of the maximum shear stress decreases linearly with a decreasing shear layer growth rate and decreasing mean flow density.

Plane mixing layers have growth rates of $d \delta / d x \approx 0.16$, which suggests that $\tau_{\mathrm{Max}} / \rho \Delta U^{2} \approx 0.027$. This estimate is nearly twice the peak measured Reynolds stresses $\tau$ $=-\overline{u^{\prime} v^{\prime}} / \Delta U^{2} \approx 0.13$ (Spencer and Jones ${ }^{32}$ ). With $d \delta / d x$ $\approx 0.25, \tau_{\mathrm{Max}} / \rho \Delta U^{2} \approx 0.04$. This suggests that the maximum value of the Reynolds stress $\left(-\overline{u^{\prime} v^{\prime}} / U_{\mathrm{fs}}^{2}\right)_{\mathrm{Max}} \approx 0.02 \cdot(\Delta U /$ $\left.U_{\mathrm{fs}}\right)^{2}$. If $\Delta U$ is the measured velocity difference across the layer, $\left(-\overline{u^{\prime} v^{\prime}} / U_{\mathrm{fs}}^{2}\right)_{\mathrm{Max}} \approx 0.002$, and this is about an order of magnitude smaller than the measured value of between 0.02 and 0.03 . However, in the present experiment, the high-speed flow essentially drives a recirculating low speed flow in the manner similar to a rearward facing step, where $\Delta U \approx U_{\mathrm{fs}}$. With this assumption, $\left(-\overline{u^{\prime} v^{\prime}} / U_{\mathrm{fs}}^{2}\right)_{\mathrm{Max}} \approx 0.02$, which is close to the values measured here.

The above relationship can be used to determine trends in the shear stress with changes in the extent of cavitation within the shear layer. As the cavitation number decreases, the mean density in the cavitating shear layer decreases by less than $2 \%$, and no significant variation was observed in the growth rate, although there is considerable uncertainty in this measurement. The mean profiles in the region just downstream of the cavitation suggest that $\Delta U$ in region $\mathrm{B}$ increases by about $10 \%$ at the lowest cavitation number. A reduction in mean density would suggest a reduction in the shear stresses in the cavitating layer, while an increase in the strain rate within the layer would suggest an increase. An examination of the Reynolds stresses in Fig. 17 indicates that the advent of cavitation reduced the stresses by about a factor of 2 , and this is accompanied by a reduction of cross-stream fluctuations shown in Fig. 16. The changes in mean density or growth rate do not account for such a difference, and the changes in the strain rate across the layer suggest an increase in shear stress with cavitation.

Reduction in the Reynolds stresses is most pronounced for the case of $\sigma=0.50$. This is the condition when the cavitation has developed the most without the formation of a large bubbly mixture beneath the cavity. For the case of $\sigma$ $=0.45$, the flow beneath the shear layer is full of large vapor bubbles that are trapped and recirculated [see Figs. 5(d) and $5(\mathrm{e})$, for example]. It is possible that the extent of the shear across the shear layer is reduced by the presence of this vapor. This could explain why the trend reverses with a further decrease in cavitation number below 0.50 .

\section{CONCLUSIONS}

Visual observations of the shear layer suggest that the overall formation, growth, and convection of the primary and secondary vortical structures in the shear layer are not significantly affected by the presence of cavitation. The average flow field downstream of the cavitating shear layer is not significantly altered both in terms of average velocities and mean pressure drop across the test section.

The largest differences between the cavitation and the noncavitating flows were in the turbulent fluctuations examined downstream of the cavitating shear layer. The streamwise fluctuations increased by about $15 \%$ compared to the noncavitating case in the center of the shear flow. However, the maximum cross-stream fluctuations and Reynolds stresses decreased by about 30\%. As bubbles collapse, an increase in turbulence levels is expected (Laberteaux and Ceccio $\left.{ }^{5,6}\right)$. The decrease in the cross-stream fluctuations and Reynolds stresses suggest that the cavitation within the cores of streamwise vortices has decreased the coupling between the streamwise and cross-stream velocity fluctuations. This is consistent with the hypothesis of Belahadji et al. ${ }^{22}$ whereby cavitation in the cores of the streamwise vortices decoupled the stretching and rotation rate of these flow structures. The reduction in Reynolds stresses downstream of the shear layer is inconsistent with the scaling of the shear stress within the shear layer based on the shear layer growth rate, mean den- 
sity, and the mean flow profiles. As these measurements have limited spatial resolution, it may be necessary to examine turbulent fluctuation at smaller scales to resolve this issue.

The production and distribution of turbulent fluctuations seem to have been significantly altered by the presence of developed cavitation. It is problematic to interpret the data from this experiment in the context of a true mixing layer. Moreover, we are left to infer the flow processes within the cavitating layer from observations of the noncavitating flow downstream. It may be necessary to examine the turbulent cavitating flow directly to resolve the questions regarding the shear stress distribution and Reynolds stresses. However, the mean shear and Reynolds stress distribution in turbulent flows can be substantially altered when the rheological behavior of the flow is altered. An example is the addition of high surfactants to a turbulent shear flow. Warholic et al. ${ }^{33}$ demonstrated that the addition of relatively small quantities of surfactants resulted in the substantial suppression of crossstream velocity fluctuations and Reynolds stresses in a turbulent channel flow. This resulted in a substantial reduction in the shear stress at the wall of the channel. Similarly, researchers have noted that the addition of microbubbles will lead to the reduction of wall shear stresses, and dynamic shear stress measurements at the wall suggest that the bubbles are also altering the dynamics of the turbulent flow (Merkle and Deutsch ${ }^{34}$ ). It is therefore possible that the presence of the cavitation within the shear layer modifying the distribution of mean shear stress across the layer through a change in the effective rheology of the flow.

Cavitating wakes have been significantly modified by developed cavitation, especially with respect to the rate of vortex shedding. Such effects were not observed here. The void fraction of the cavitating shear layer was, at most, $2 \%$. It is unclear if the void fraction in the separated region behind the bluff bodies is significantly higher, but this may be the case. Moreover, the dynamics of the shedding process behind bluff object is altered by changes in the pressure field near the bluff body. The presence of developed cavitation can alter the overall pressure field, especially if the cavitating object is confined in a flow channel. In the present study, the presence of the cavitation did not significantly change the average pressure field.

Last, these results suggest that a single-phase simulation of shear flow may be used to determine the extent of the cavitation within the shear flow. A method is needed that can successfully simulate the strongest vortices in the flow over a range of scales. While such single-phase simulations are, at present, difficult to conduct at high Reynolds numbers, methods such as large eddy simulations may eventually be used to reveal the largest vortical structure in a flow field. Once identified, the extent of the cavitation can be estimated. This is particularly true of incipient cavitation where the influence on the underlying shear flow may be neglected.

\section{ACKNOWLEDGMENT}

Financial support for this work was provided by the Office of Naval Research through Contract No. N00014-960076, Dr. E. P. Rood, Technical Monitor.
${ }^{1}$ C. E. Brennen, Cavitation and Bubble Dynamics (Oxford University Press, Oxford, 1995).

${ }^{2}$ K. L. Laberteaux and S. L. Ceccio, "Flow in the closure region of closed partial attached cavitation," 3rd International Symposium on Cavitation, April 1998, Grenoble, France, p. 197.

${ }^{3}$ K. Laberteaux, S. L. Ceccio, V. Mastrocola, and J. Lowrance, "High speed digital imaging of cavitating vortices," Exp. Fluids 24, 489 (1998).

${ }^{4}$ S. Gopalan and J. Katz, "Flow structure and modeling issues in the closure region of attached cavitation," Phys. Fluids 12, 895 (2000).

${ }^{5}$ K. R. Laberteaux and S. I. Ceccio, "Partial cavity flows: Part 1-Cavities forming on models without spanwise variation," J. Fluid Mech. 431, 1 (2000).

${ }^{6}$ K. R. Laberteaux and S. L. Ceccio, "Partial cavity flows: Part 2-Cavities forming on test objects with spanwise variation," J. Fluid Mech. 431, 43 (2000).

${ }^{7}$ Y. Kawanami, H. Kato, and H. Yamaguchi, "Three-dimensional characteristics of the cavities formed on a two-dimensional hydrofoil," in Ref. 2.

${ }^{8}$ M. Callenaere, J. P. Franc, and J. M. Michel, "Influence of cavity thickness and pressure gradient on the unsteady behavior of partial cavities," in Ref. 2.

${ }^{9}$ G. L. Brown and A. Roshko, "On density effects and large structure in turbulent mixing layers," J. Fluid Mech. 64, 775 (1974).

${ }^{10}$ C. D. Winant and F. K. Browand, "Vortex pairing: The dynamics of turbulent mixing layer growth at moderate Reynolds numbers," J. Fluid Mech. 63, 237 (1974)

${ }^{11}$ L. P. Bernal, R. E. Breidenthal, G. L. Brown, J. H. Konrad, and A. Roshko, "On the development of three-dimensional small scales in turbulent mixing layers," 2nd International Symposium on Turbulent Shear Flows, 1981, p. 305.

${ }^{12}$ J. C. Lasheras, J. S. Cho, and T. Maxworthy, "On the origin and evolution of streamwise vortical structures in a plane, free shear layer," J. Fluid Mech. 172, 231 (1986).

${ }^{13}$ J. C. Lasheras and H. Choi, "Three-dimensional instability of a plane free shear layer: An experimental study of the formation and evolution of streamwise vortices," J. Fluid Mech. 189, 53 (1988).

${ }^{14}$ R. E. A. Arndt, "Cavitation in fluid machinery and hydraulic structures," Annu. Rev. Fluid Mech. 13, 273 (1981).

${ }^{15}$ J. Katz, "Cavitation phenomena within regions of flow separation," J. Fluid Mech. 140, 397 (1984).

${ }^{16}$ J. Katz and T. J. O'Hern, "Cavitation in large scale shear flow," Trans. ASME J. Fluids Eng. 108, 373 (1986).

${ }^{17}$ T. J. O'Hern, “An experimental investigation of turbulent shear flow cavitation," J. Fluid Mech. 215, 365 (1990).

${ }^{18}$ R. W. Kermeen and B. R. Parkin, "Incipient cavitation and wake flow behind sharp-edged disks," California Institute of Technology Hydrodynamics Laboratory Report No. 85-4, 1957.

${ }^{19}$ J. O. Young and J. W. Holl, "Effects of cavitation on periodic wakes behind symmetric wedges," J. Fluid Mech. 63, 237 (1966).

${ }^{20}$ R. E. A. Arndt, "Investigation of the effects of dissolved gas and free nuclei on cavitation and noise in the wake of a sharp-edged disk," Joint IAHR/ASME/ASCE Symposium on Fluid Machinery, Fort Collins, CO, 1978.

${ }^{21}$ R. E. A. Arndt and W. K. George, "Pressure fields and cavitation in turbulent shear flows," 12th Symposium on Naval Hydrodynamics (National Academy, Washington, DC, 1979).

${ }^{22}$ B. Belahadji, J. P. Franc, and J. M. Michel, "Cavitation in the rotational structures of a turbulent wake," J. Fluid Mech. 287, 383 (1995).

${ }^{23}$ D. L. George and S. L. Ceccio, "Cavitation and multiphase flow laboratory at the University of Michigan," 24th American Towing Tank Conference, edited by P. Johnson, 1995.

${ }^{24}$ I. Wygnanski and H. E. Fiedler, "The two-dimensional mixing region," J. Fluid Mech. 41, 327 (1970)

${ }^{25}$ P.-W. Yu and S. L. Ceccio, "Bubble populations downstream of an attached cavity," J. Fluid Mech. 119, 782 (1997).

${ }^{26} \mathrm{M}$. A. Hernan and J. Jimenez, "Computer analysis of a high-speed film of the plane turbulent mixing layer," J. Fluid Mech. 119, 323 (1982).

${ }^{27} \mathrm{~J}$. Jimenez, "A spanwise structure in the plane shear layer," J. Fluid Mech. 132, 319 (1983).

${ }^{28}$ J. Jimenez, M. Cogollos, and L. P. Bernal, "A perspective view of the plane mixing layer," J. Fluid Mech. 152, 125 (1985)

${ }^{29} \mathrm{~L}$. Briançon-Marjollet and L. Merle, "Inception, development and noise of 
a tip vortex cavitation," 21st Symposium on Naval Hydrodynamics, Trodheim, Norway, 1996.

${ }^{30}$ R. E. A. Arndt and A. P. Keller, "Water quality effects on cavitation inception in a trailing vortex," J. Fluids Eng. 114, 430 (1992).

${ }^{31}$ J. C. Hermanson and P. E. Dimotakis, "Effects of heat release in a turbulent, reacting shear layer," J. Fluid Mech. 199, 333 (1989).

${ }^{32}$ B. W. Spencer and B. G. Jones, "Statistical investigation of pressure and velocity fields in the turbulent two stream mixing layer," 11th Aerospace Sciences Meeting, 1971, AIAA Paper 71-613.

${ }^{33}$ M. D. Warholic, G. M. Schmidt, and T. J. Hanratty, "The influence of a drag-reducing surfactant on a turbulent velocity field," J. Fluid Mech. 388, 1 (1999).

${ }^{34}$ C. L. Merkle and S. Deutsch, "Microbubble drag reduction in liquid turbulent boundary layers," Appl. Mech. Rev. 45, 103 (1992). 